\title{
LYMPHOVENOUS ANASTOMOSIS FOR THE TREATMIENT OF LYMPHEDEMA: A SYSTEMATIC REVIEW OF THE LITERATURE AND MIETA-ANALYSIS
}

\author{
E. Nacchiero, M. Maruccia, R. Elia, F. Robusto, G. Giudice, O.J. Manrique, \\ C.C.C.ampisi, G. Giudice
}

Division of Plastic and Reconstructive Surgery (EN,MM,RE,FR,GG), Department of Emergency and Organ Transplantation, University of Bari, Bari, Italy; ICLAS, GVM Care \& Research (CCC), Rapallo, Genova, Italy; Salus Hospital, GVM Care \& Research, Reggio Emilia, Italy; London School of Economics (GG), Department of Statistics, London, United Kingdom; Division of Plastic and Reconstructive Surgery (OJM), Mayo Clinic, Rochester, Minnesota, United States

\section{ABSTRACT}

Lymphovenous anastomosis (LVA) has been described as an effective treatment for early stages of lymphedema (LE). The aim of this study was to deepen the evaluation of the effectiveness of $L V A$ by performing a metaanalysis to provide information about its utility in specific anatomical sites, clinical stages, duration of lymphedema, and surgical technique. A systematic literature search using PubMed/Medline, Google Scholar, and Cochrane Database was performed in November 2019. Only original studies in which exclusively LVA was performed for primary and/or secondary lymphedema in humans were eligible for data extraction. A meta-analysis was performed on articles with a well-defined endpoint and a subgroup analysis was conducted in relation to surgical technique, duration of lymphedema, stage of pathology. Forty-eight studies, including 6 clinical trials and 42 lowrisk bias observational studies were included in our meta-analysis. 1,281 subjects were included and the majority of articles reported a pre-post analysis. Lymphaticovenular anastomosis appears to result effectively in treatment of lymphedema with an odds ratio of 0.07 (CI: $0.04,0.13, p<0.001$ ). All subgroup metaanalyses were statistically significant for $L V A s$ specifically with regard to anatomical site, clinical stage, duration of $L E$, or type of microsurgical procedure $(p<0.05)$.

Our meta-analysis confirmed the efficacy of LVAs for the treatment of lymphedema, even when subgroup analysis was performed for clinical stage, duration of pathology, anatomical site of lymphedema, or type of microsurgical procedure. Further prospective trials with a common clearly defined outcome measure are warranted for an unbiased evaluation.

Keywords: LVA, lympho-venous anastomosis, lymphedema, treatment, lymphatics, metaanalysis

\section{Lymphedema (LE) is a condition} characterized by accumulation of lymphatic fluid in the interstitial tissue of the arms, legs, and occasionally other parts of the body (1). It is the result of an impairment to the outflow of lymphatic fluid from the affected area and accumulating lymphatic fluid is responsible for the consequent inflammation, lipogenesis, fibrosis, infections, and elephantiasis (2). LE may be primary - due to dysplasia of lymphatic vessels or valvular dysfunction - or secondary to infection, surgical lymphadenectomy, and/or radiotherapy (3). Although worldwide the most common cause of LE is filariasis, the 
most frequent etiology in developed countries is related to cancer and its treatment (4-6). The International Society of Lymphology established a staging system for lymphedema into 4 clinical classes (7); Chang et al developed a classification based on ICG lymphangiography findings (8), while Campisi et al introduced a classification that combines clinical presentation and lymphoscintigraphic patterns (9). Correct lymphedema staging is an important tool for the management of LE and a guide to better therapeutic options. Although surgical procedures for prevention or treatment of LE have been already widely described, the majority of patients are still managed non-operatively, reserving surgery to those who are dissatisfied (10). Non-surgical procedures include manual lymphatic drainage, compression therapy with low-stretch bandages, skin care, and exercises (11). While the effectiveness of these approaches have been documented, the success of these procedures requires an intense training of therapists and patients as well as continued maintenance for the rest of a patient's life (12). Surgery could overcome these limits by improving the physiological circulation of lymphatic fluid in progressive and resistant lymphedema. A variety of procedures have been proposed in the past decades, but modern accepted surgical techniques for treating lymphedema include liposuction, vascularized lymph node transfer, and lymphovenous anastomosis (LVA) (13-18). The effectiveness of these procedures has been reported in previous studies, but the literature demonstrates a great heterogeneity in presented outcomes. Specifically, while measurement of limb circumference is the most commonly used method to evaluate LE, other tests are available such as volume assessment based on computer tomography (CT), lymphography, ICG-lymphoscintigraphy, US evaluation, magnetic resonance imaging (MRI), measurement of water displacement, or condition-specific quality of life assessment tools $(19,20)$.

LVA has been well described as an effective treatment for early stage lymphedema of the extremities demonstrating a very low risk of complications and the possibility to be performed under local anesthesia (21-24). Nevertheless, there is no consensus on the best surgical technique or how to measure surgical outcome and effectiveness of the procedure. There are meta-analyses available in literature addressed to the advantages and disadvantages of LVAs in treatment of lymphedema, but they include only few available clinical trials and enrolled patients only with a specific site or etiology of the lymphedema and with-out appropriate subgroup analysis $(25,26)$.

The aim of this study was to evaluate the effectiveness of LVA for the treatment of lymphedema by performing a meta-analysis with subgroup evaluations to provide information about its utility in specific anatomical sites, stages of lymphedema, duration of pathology, and also examining the different surgical techniques.

\section{MATERIALS AND METHODS}

\section{Selection of Studies}

A literature review was performed according to the Preferred Reporting Items for Systematic Reviews and Meta-Analyses (PRISMA) statement. In November 2019, an electronic search was conducted through PubMed/Medline, Google Scholar, and Cochrane Database restricted to the English language. The databases were searched using the following set of search Medical Subject Headings (MeSH) terms including 'lymphedema', 'lymphatic venous', 'lympho-venous', 'lymphaticovenular', 'anastomosis', 'bypass', 'shunt', 'lymphoplasty', in combination with the Boolean logical operators (AND or OR). Only original studies in which exclusively LVA was performed for the treatment of primary and/or secondary lymphedema in humans were eligible for data extraction. Studies on filariasis-related lymphedema, on preventive techniques, or on lymph nodes transfers were excluded from the review. No limitation was placed on the number of patients included in the studies. Data extraction was performed by two independent reviewers (EN and FR) identifying relevant 
articles for retrieval. Relevant data was determined prior to reading selected articles. For all included studies, we documented the type of study, year of publication, authors, number of patients, number of anastomosis, primary endpoints, type of microsurgical technique, duration of lymphedema prior to surgery, stage of lymphedema, follow-up period, complications, and presence of a control group. A meta-analysis was performed on articles with a well-defined end-point. Papers published by the same research group and reporting duplicate data were excluded. Both retrospective comparative studies and retrospective case series and prospective studies were included in this review and metaanalyses. Two independent reviewers (EN and FR) reported extracted data in a spreadsheet that included relevant information. In case of divergent opinions, another independent investigator (MM) was requested to help reach a consensus. The methodological index for non-randomized studies (MINORS) was used to assess the methodological quality or risk of bias for nonrandomized studies (27). Analysis on effectiveness of LVA on LE treatment was performed both in the few published controlled clinical trials and in high quality non-randomized studies. Moreover, additional analysis of subgroups was performed to com-pare whether LVA was more effective accord-ing to the surgical technique, duration of lymphedema prior to surgery, and stage of pathology. Given that included studies used different types of classification systems, the methodology used in the current study to uniform the different classification systems can be found in Table 1 (28).

TABLE 1
Uniform Stage Classifications of
Lymphedema developed for the Meta-
Analysis and the Associated Notations by
the Campisi, ISL and Cheng Scales
\begin{tabular}{|l|c|c|c|} 
Stage & Campisi & ISL & Cheng \\
\hline Early & I & $0-1$ & $0-1$ \\
\hline Moderate & II - III & 2 & 2 \\
\hline Severe & IV - V & $3-4$ & $3-4$ \\
\hline
\end{tabular}

In the following sections, we focus our attention on the difference between two groups with respect to a binary outcome. Specifically, we employ odd ratios to make the results uniform. The odds for a group is defined as the ratio of the number of patients in the group who achieve the stated endpoint and the number of patients who don't. We treat an increase in the reference index - for example, volumetry - as the main event. This means that if the majority of patients experience a reduction in the volumetry, the odd ratio will be smaller than 1 . A ratio of 1 indicates no difference - that is, the outcome is just as likely to occur in the control group as it is in the treatment group.

\section{Statistical Analysis}

When pooling study results, two main approaches can be used depending on the effect size heterogeneity: the Fixed-Effect Model or the Random Effect Model. Under the first model, the main assumption is that all results originate from a single homogeneous population. By contrast, with the latter, we assume that the true effect distribution varies from study to study. I-square was used to calculate heterogeneity among the studies. A probability value of I-square $\geq 50 \%$ indicated the presence of significant heterogeneity. The fixed effects model was used in the presence of no significant inter-study heterogeneity; otherwise, the random effects model was used. Dichotomous variables were pooled by the Mantel-Haenszel method and compared using Odds Ratio with 95 percent confidence intervals. Continuous variables were analyzed with the inverse variance method, using weighted mean differences with 95 percent confidence intervals. A p-value $<\mathbf{0 . 0 5}$ was considered statistically significant. Subgroups analysis dwell in two main parts: (1) pooling the effect within each subgroup, and (2) analyzing the effects between the subgroups. This latter aggregation is performed using a fixed or random effect according to heterogeneity measures. The Begg and Mazumdar test (using Kendall's method) was used in analysis involving a small number of studies; otherwise, the Egger test was used to 
detect publication biases. Values of $\mathrm{p}<\mathbf{0 . 0 5}$ indicated presence of significant biases among studies. All statistical analyses were performed using SAS Software Release 9.4 (SAS Institute, Cary, NC).

\section{RESULTS}

\section{Studies Included in Meta-Analysis}

A total of 1221 records were retrieved (Fig. 1). After removal of duplicates, the literature search identified 916 studies. Case reports, studies on animals, non-original articles, and studies on preventive LVAs were excluded, identifying 99 eligible full-text articles. After full-text screening, additional 37 articles were excluded by further analysis because of inadequate report of cases or results, simultaneous use of LVAs and lymph node transfers or grafts, or presence of duplicated data in different papers. Of the remaining 62 studies, 6 clinical trials (29-34) were included in meta-analysis, while 58 observational studies were subjected to a further qualitative analysis using MINOR index to quantify the risk of bias (Table 2). The global ideal MINOR index score was 16 for noncomparative studies and $\mathbf{2 4}$ for comparative studies. We considered comparative studies with a MINOR index score $>\mathbf{2 0}$ and noncomparative studies with a MINOR index score $>12$ as low risk of bias (35). Using these criteria, 14 observational studies (36-49) were excluded for high risk of bias, while 42 low-risk of bias studies were included in current meta-analysis.

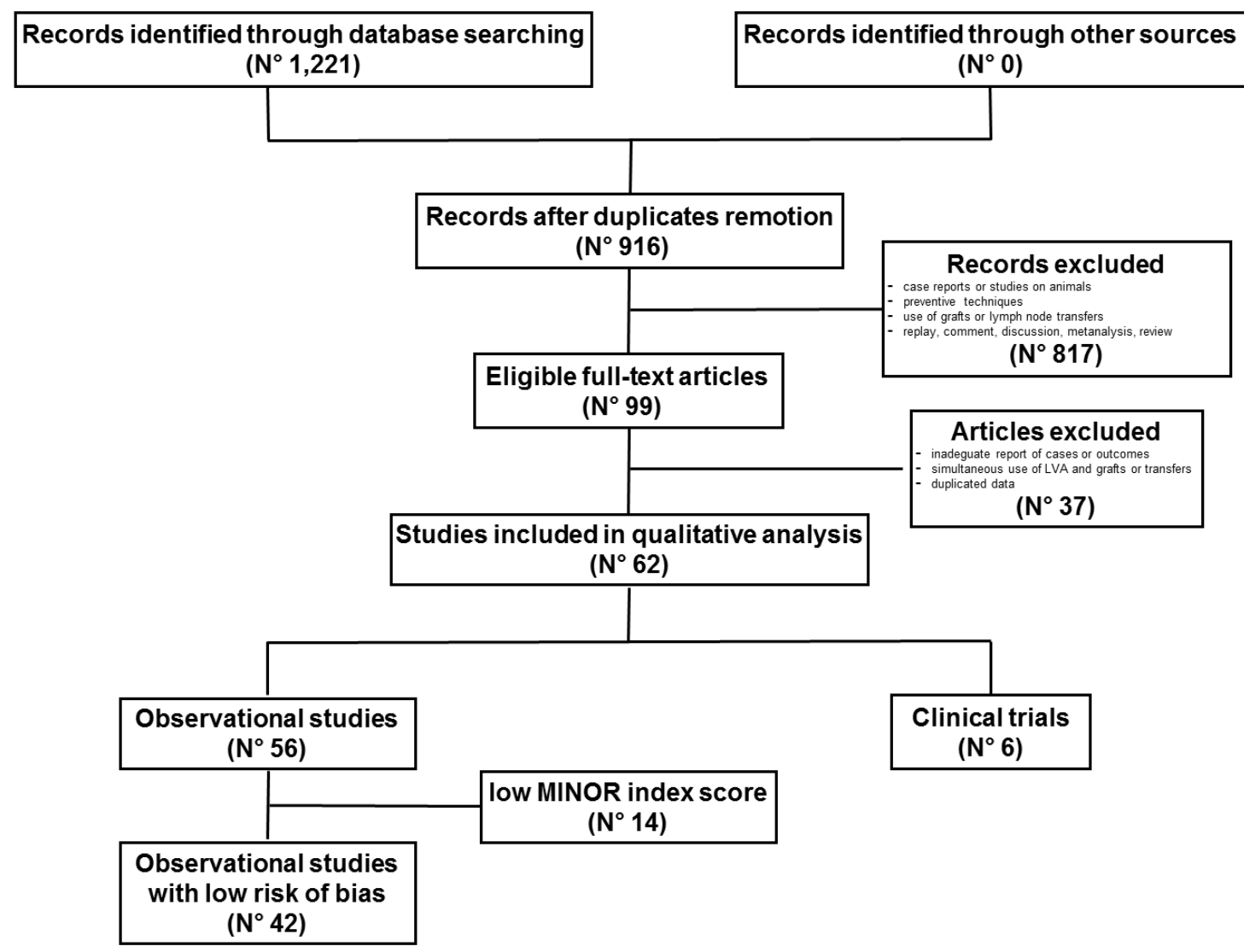

Fig. 1. PRISMA algorithm detailing the selection of studies for analysis. 


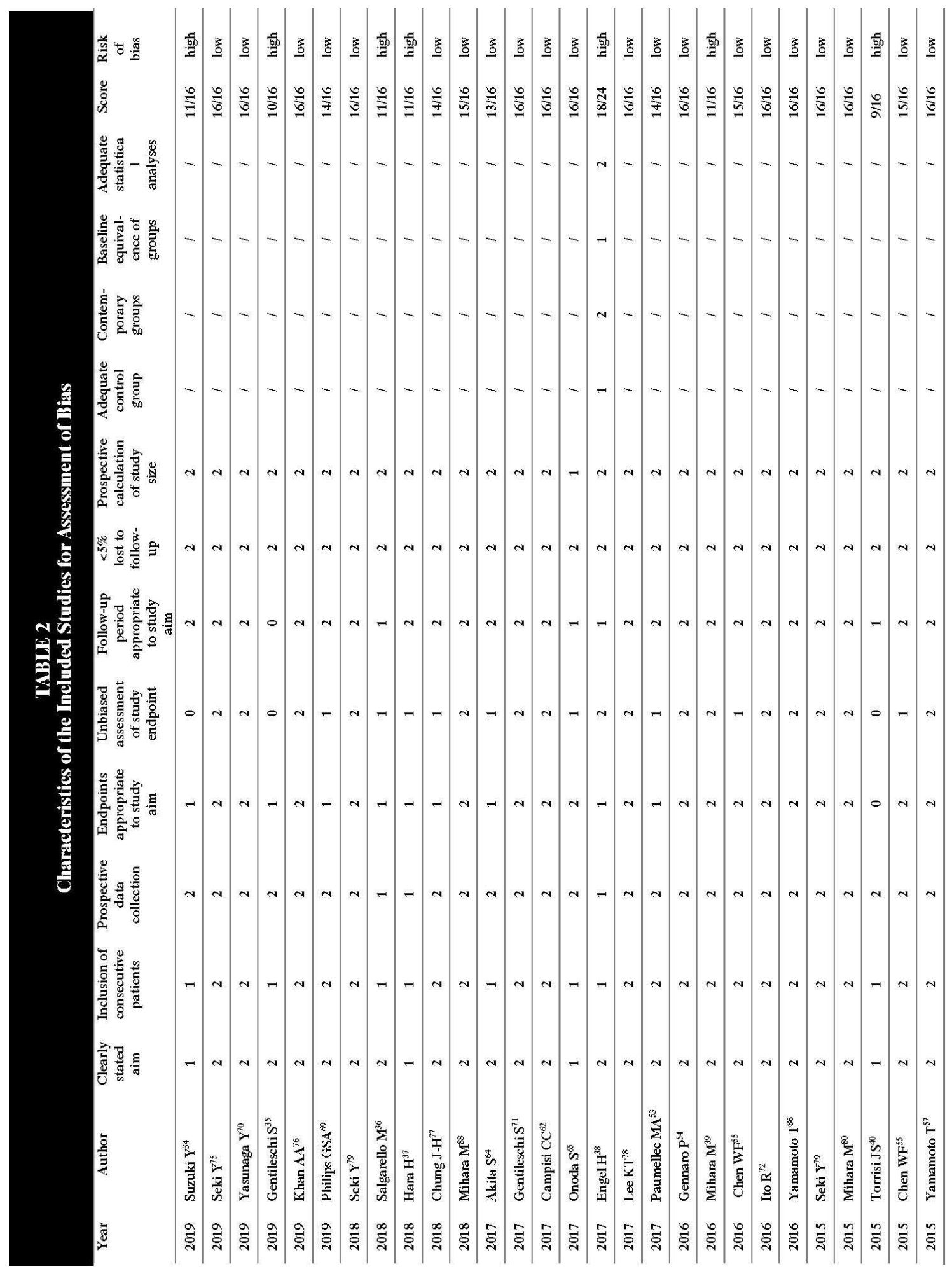




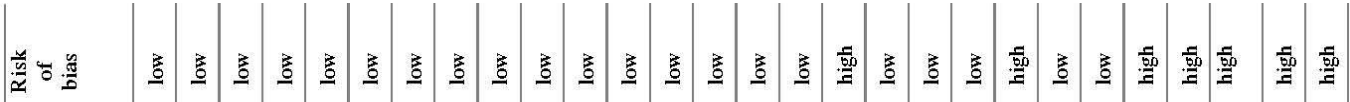

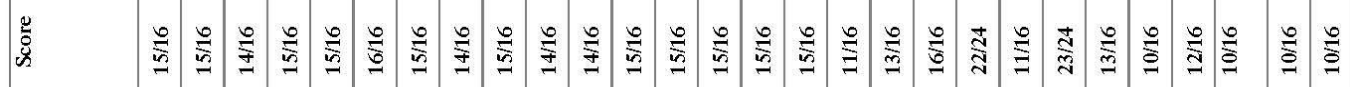

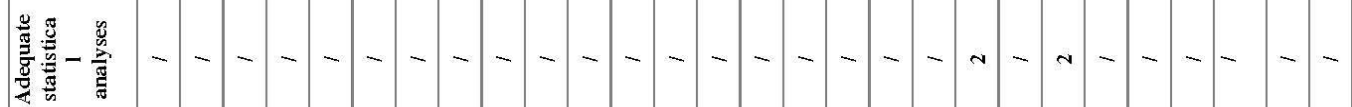

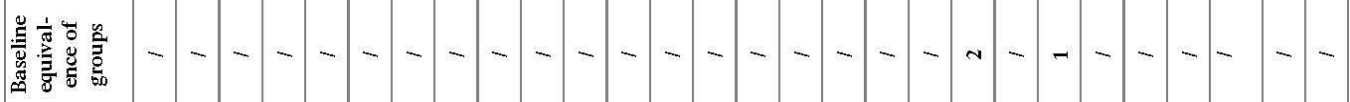

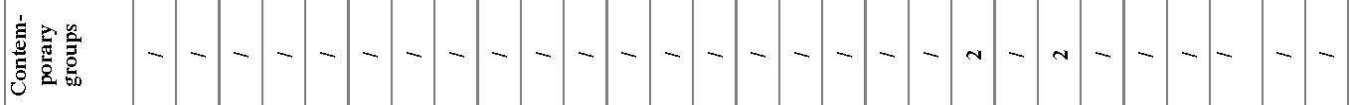

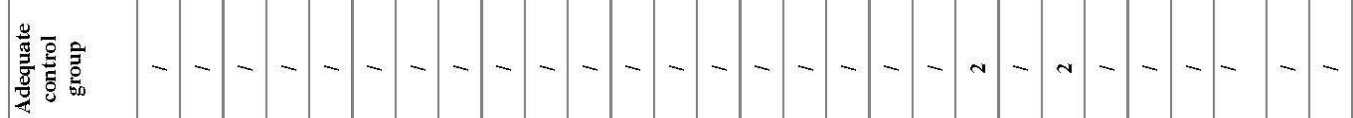

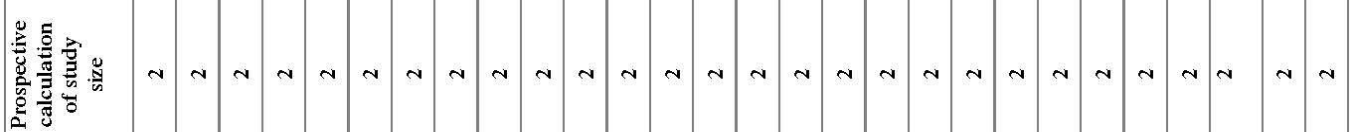

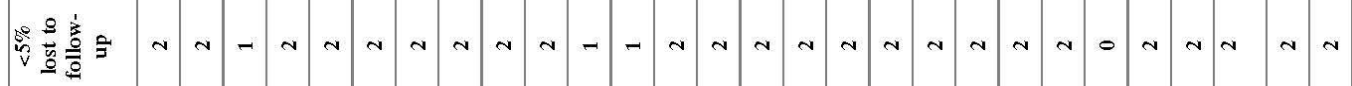

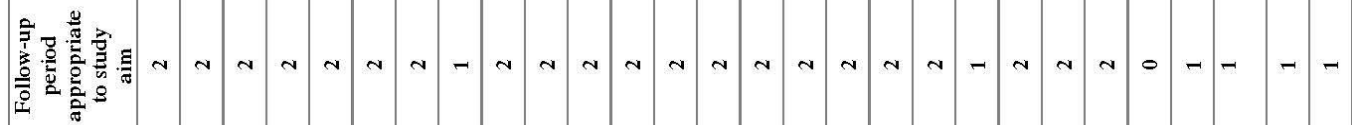

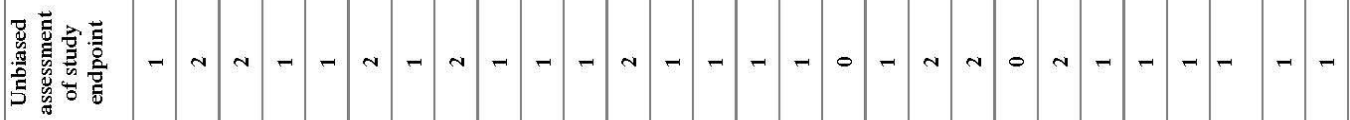

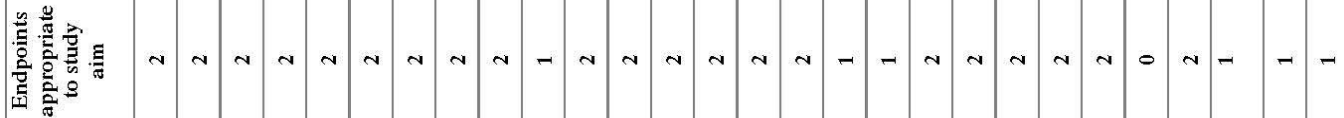

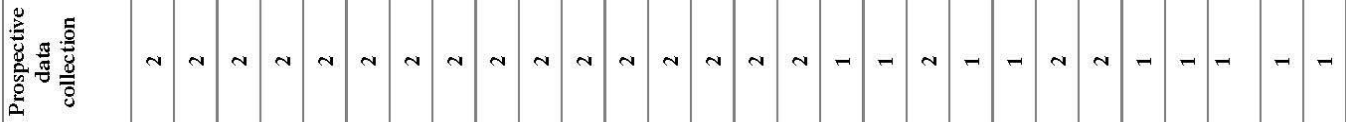

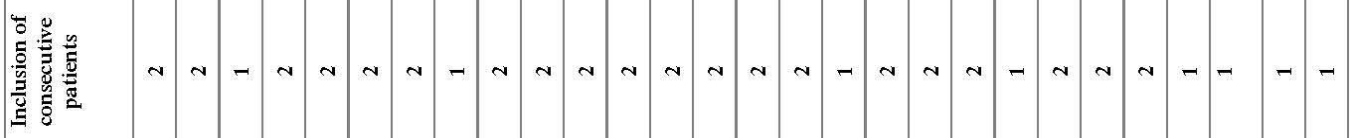

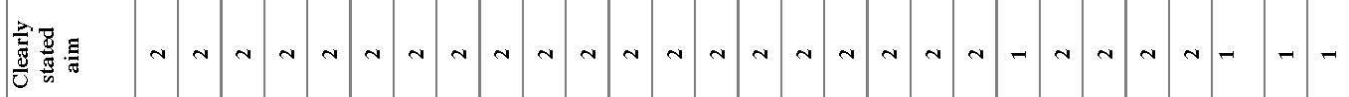

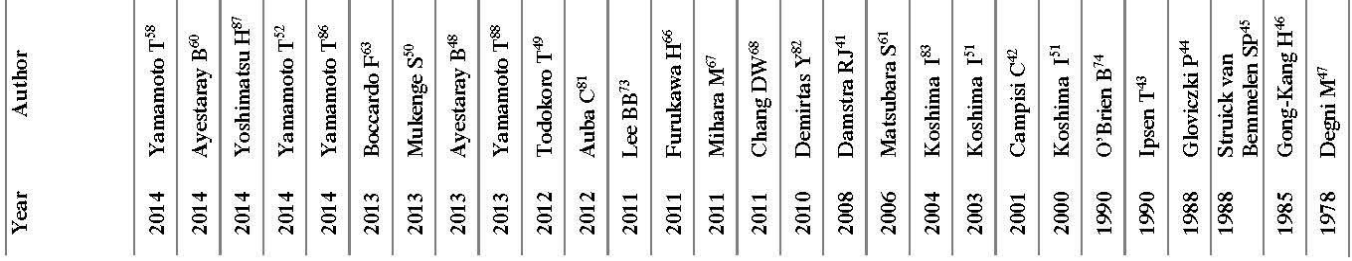


Characteristics of Studies Included in MetaAnalysis

Overall, 48 studies reporting the use of LVAs for the treatment of lymphatic disorders were analyzed: 6 clinical trials and 42 low-risk of bias observational studies (Table 3). The majority investigated the treatment of upper and lower limbs LE, while only 3 studies reported the effect of LVAs on lymphedema of other fields: head and neck (50), pelvis (51), and scrotum (52). In total 1,281 subjects were included in the current analysis and the mean follow-up period ranged from 3 months to 12 years. A control group was present only in 2 studies $(53,54)$, while the majority of articles reported a pre-post analysis. The classification scales used in the staging of lymphedema were Campisi staging, the International Society of Lymphology (ISL) classification system, and Cheng's lymphedema scale. Using the methodology detailed in Table 1 to uniform the different classification systems, we classified the stage of LE into early, moderate, and severe. Almost all the patients showed an early or moderate stage of LE at diagnosis, while only 8 studies included patients with severe stage of LE (51,55-61). Regarding the technical procedure, end-to-end and side-to-end anastomosis were the most utilized, while other procedures like end-to-side anastomosis $(50,62,63)$ and multiple-lymphatic-venous anastomosis $(64,65)$ (MLVA) were reported in a low number of studies. In some studies the type of anastomosis was not specified $(42,61$, 66-71); while in others, authors performed varied types of anastomoses but they didn't specify the technique applied in each patient (29-31,72-76). The primary outcome measure used in the majority of studies was volumetry $(41,77-86)$, specifically volume or circumference reduction, while standardized and validated indices (like LEL and UEL) were used as primary outcome in others (87-91). Only few studies included in the current meta-analysis reported subjective indices such as improvement in quality of life $(57,71)$ or US/CT or lymphoscintigraphic images $(51,66)$ as primary outcomes.

\section{Results of Meta-Analysis}

Only 6 clinical trials were included in the current meta-analysis and none had a control group. These studies investigated the effectiveness of end-to-end and/or side-to-end anastomosis for the treatment of upper and lower limb LE and the primary outcome was volumetry or LEL or UEL indices. The positive outcome was reported as means or difference of means between pre and post measurements, while Shih et al (33) reported the frequency of patients with a significant volumetric improvement. Akita et al (34) showed the improvement in LEL index in patients with lymphoscintigraphy, venous reflux, and without venous reflux after LVAs separately. The pooled analyses presented a high heterogeneity (I-square $=68 \%$ ), thus a random effect model for analysis was used. The results revealed a significant positive effect of LVAs in treatment of $\mathrm{LE}(\mathrm{OR}=0.34,95 \% \mathrm{CI}=0.14$ 0.81, p $<0.01$ ) (Fig. 2). No publication bias was observed in these analyses by the Egger test $(t=0.33 ; p=0.75)$. Because of the low number of errors, which measure the uncertainty of each study, the grey squares indicate the odds ratio of each study while the area reflects the relative weight contribution of the studies when pooling the results. The horizontal line indicates their $\mathbf{9 5 \%}$ confidence interval, i.e., how many times the interval would contain the true underlying effect if the experiment is repeated multiple times. The solid vertical line $(\mathrm{OR}=1)$ indicates that there is no difference in terms of outcome with or without the treatment. If the confidence interval is containing 1 , then this difference is not statistically significant at a $95 \%$ level. The overall effect for each study is calculated as a weighted average of the individual studies and displayed as a diamond. Both the fixed effect and random effect model are displayed. In the specific case, data exhibit a high level of heterogeneity as indicated by I-square, thus a random effect model would be more appropriate. Furthermore, subgroups are also pooled according to a random effect model given the high level of I-square $=74 \%$ ). The center of the diamond 


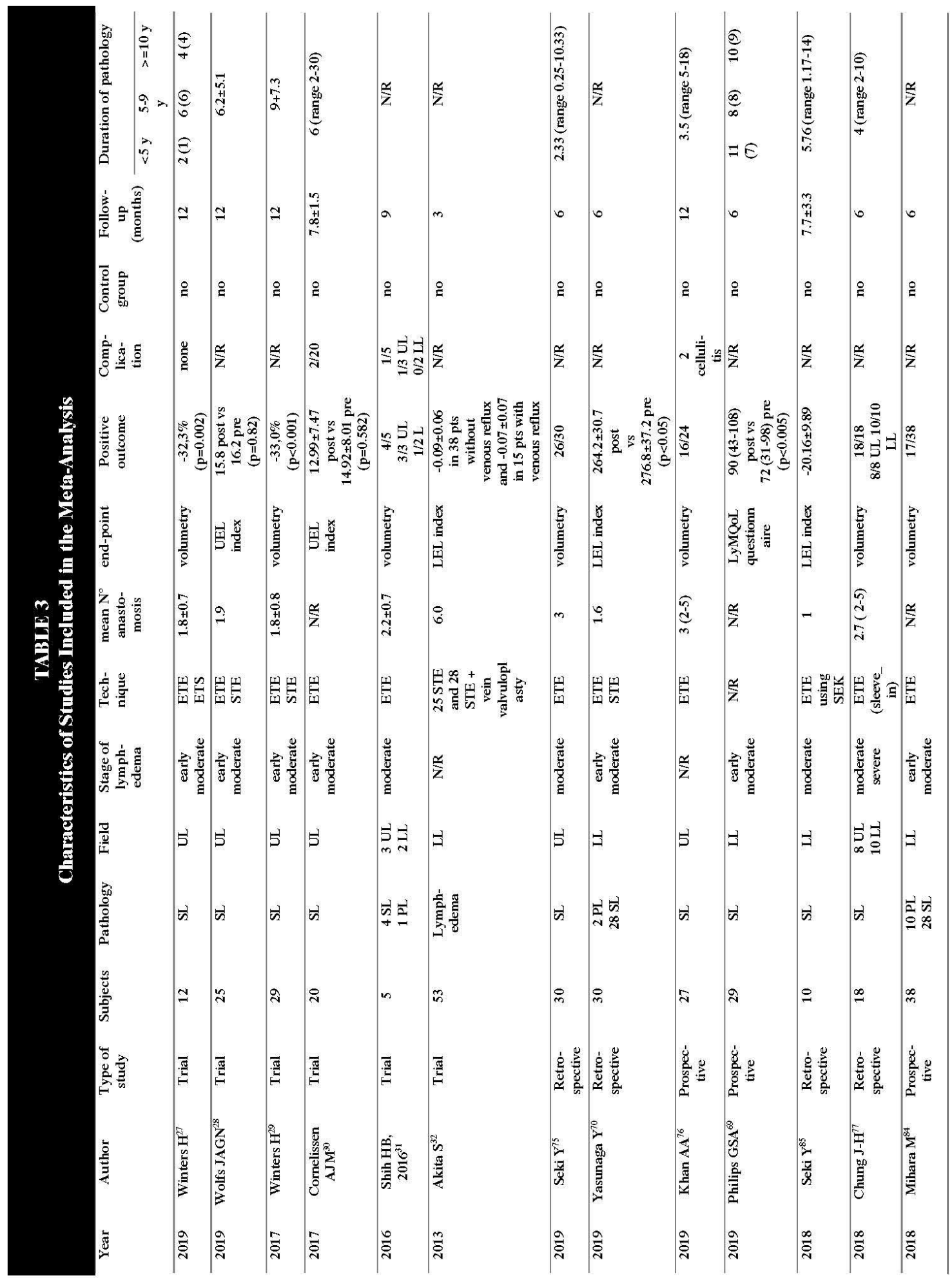




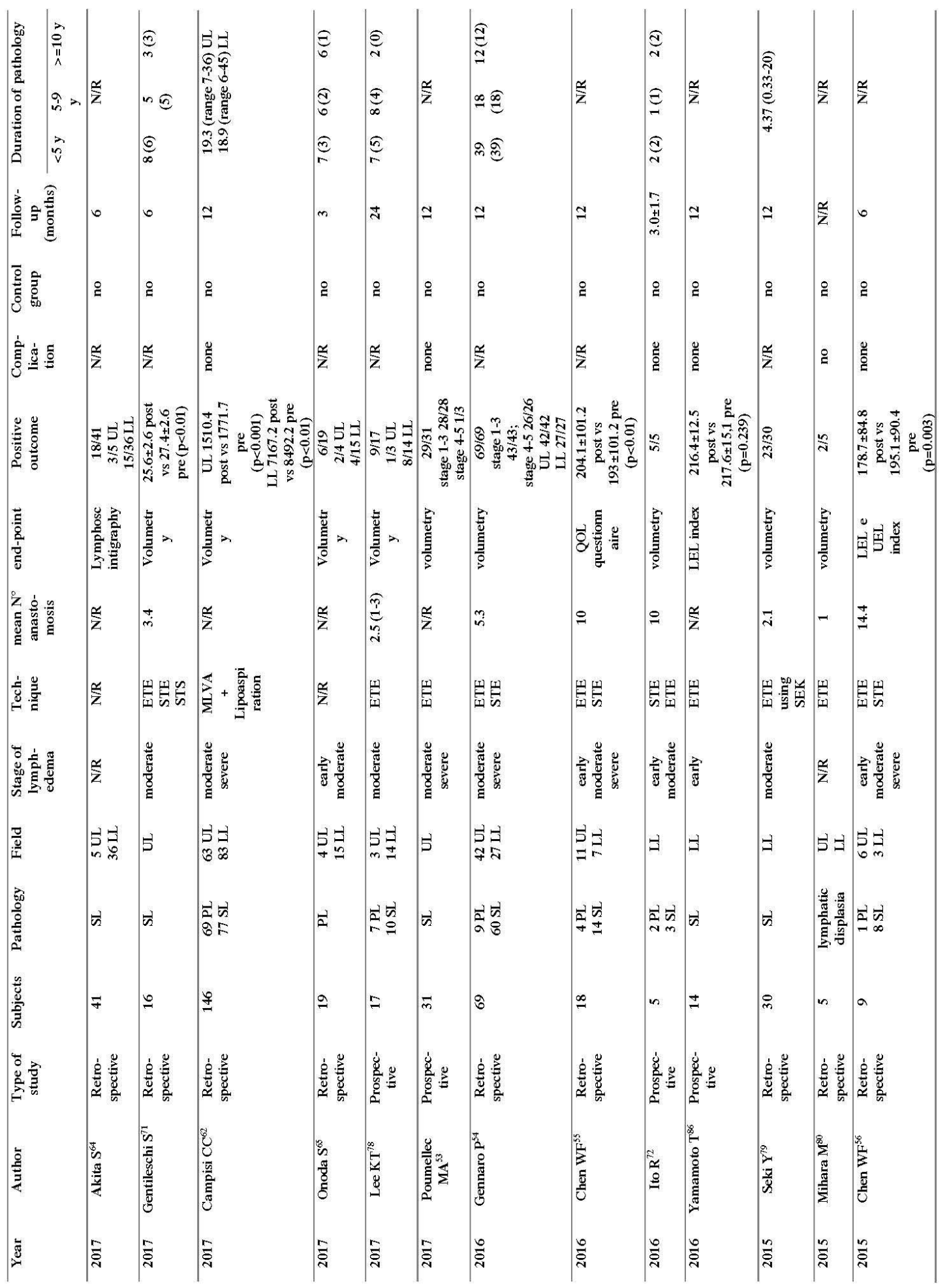




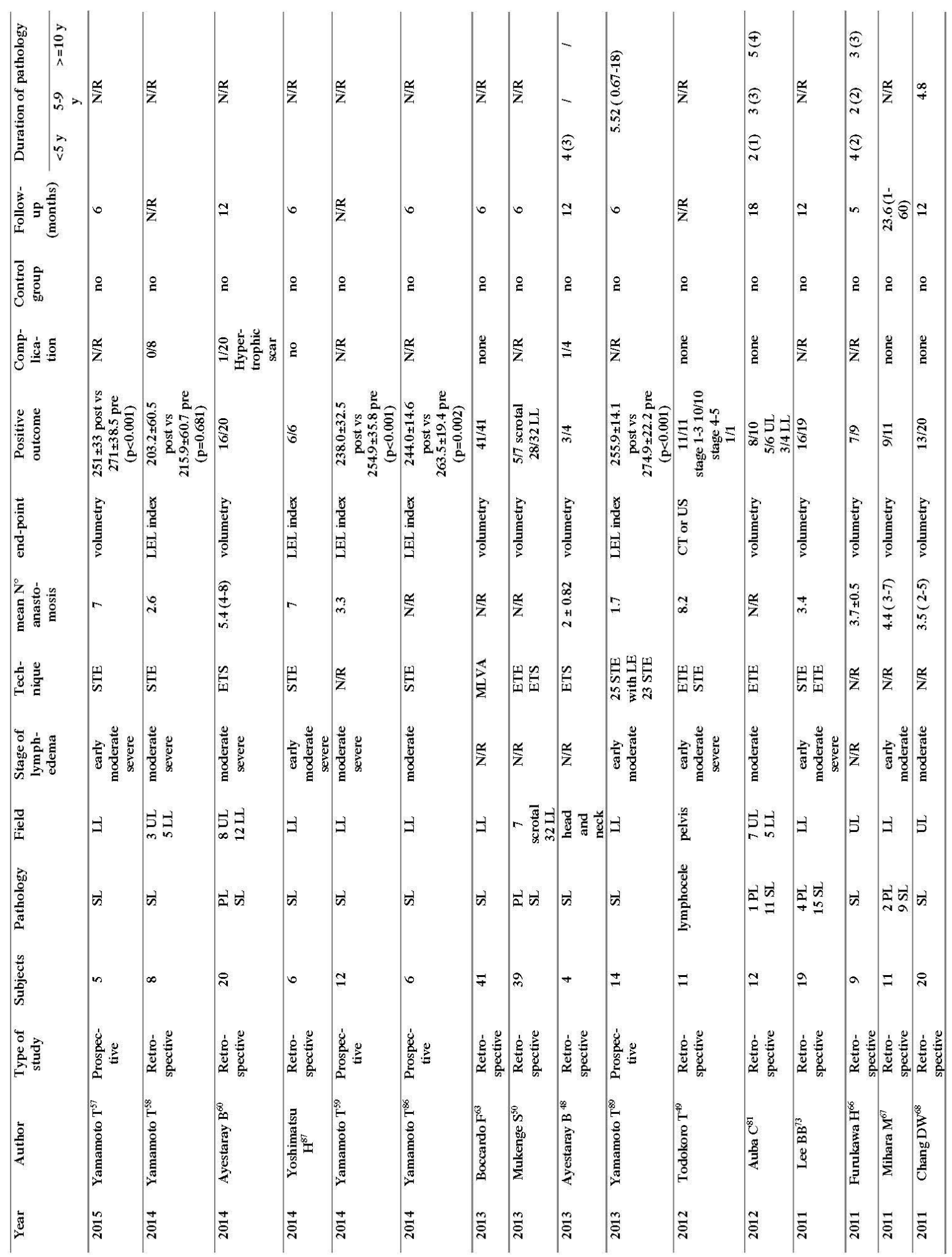




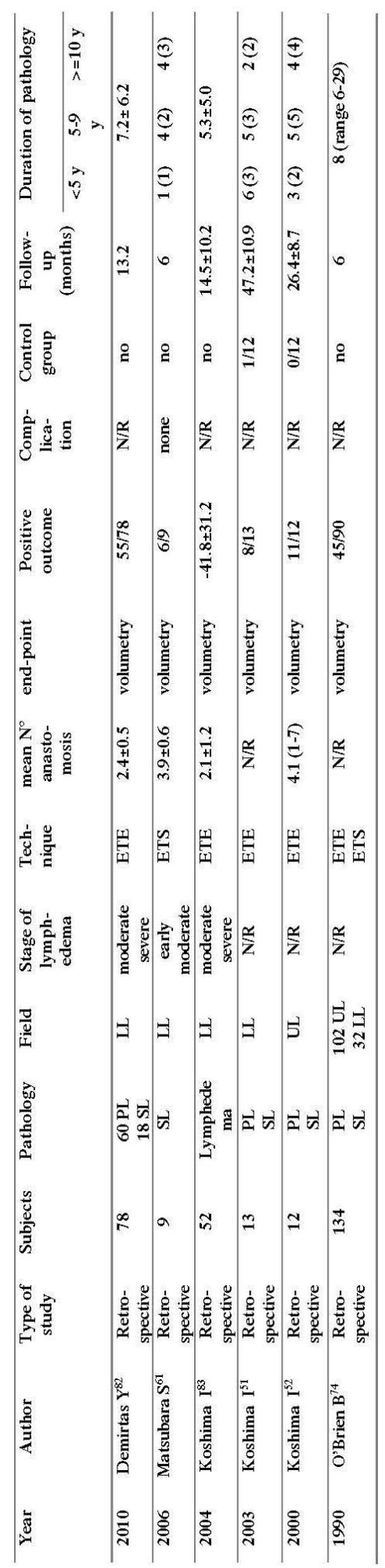

represents the combined OR of 0.07 (CI: 0.04, 0.13), at the left of the line of no-effect, meaning that fewer episodes of the outcome of interest are observed in the treatment group. Given that no part of the diamond touches the 1-line, this result is statistically significant (Fig. 2). Egger test $(\mathrm{t}=9.31, \mathrm{p}<0.01)$ testified an important publication bias, probably due to the great heterogeneity of population, LE etiology and outcomes reported in the included papers. To overcome this limit, subgroups analysis were then performed in more homogeneous cohorts, according to anatomical district, stage, duration of pathology, and microsurgical technique adopted.

The anatomic site of lymphedema was reported in all the included studies: one article reported LE in the pelvis (51), another one scrotal LE (52), one head and neck lymphedema (50), while the others show the treatment of lymphedema of the limbs. The efficacy of LVAs was testified in patients affected by lymphedema in pelvis, in which authors reported a positive outcome in all 11 patients included in the study; similar results were shown in scrotal (5/7) and head and neck (3/4) lymphedema, although a meta-analysis wasn't possible because of the lack of articles. On the other hand, results of meta-analysis in upper limbs LE in a random model effect (I-square $=70 \%$ ) testified a well-defined effect of LVAs in treatment of $\mathrm{LE}(\mathrm{OR}=0.11,95 \% \mathrm{CI}=0.05-0.26$, p<0.01) (Fig. 3, panel a). The efficacy of LVAs was evident also in meta-analysis with a random model effect (I-square $=70 \%$ ) of lower limbs LE (OR=0.08, 95\% CI=0.04-0.17, p<0.01) (Fig. 3, panel b).

The efficacy of different microsurgical techniques was evaluated in 27 papers in which the technique itself was specified. The subgroup analysis reported an efficacy for all the tested procedures: specifically, analysis using a random effect model (I-square $=80 \%$ ) demonstrated statistical significant improvement using both end-to-end anastomosis $(\mathrm{OR}=\mathbf{0 . 0 3}, \mathbf{9 5 \%} \mathrm{CI}=\mathbf{0 . 0 1 - 0 . 1 0}, \mathrm{p}<0.01)$, or sideto-end anastomosis (I-square $=70 \%, \mathrm{OR}=0.07$, 95\% $\mathrm{CI}=0.01-0.45, \mathrm{p}=0.01)$; similarly, analysis using a fixed model for MLVA (I-square $=0 \%$, $\mathrm{OR}=0.53,95 \% \mathrm{CI}=0.39-0.72, \mathrm{p}=0.04$ ) or end-to- 


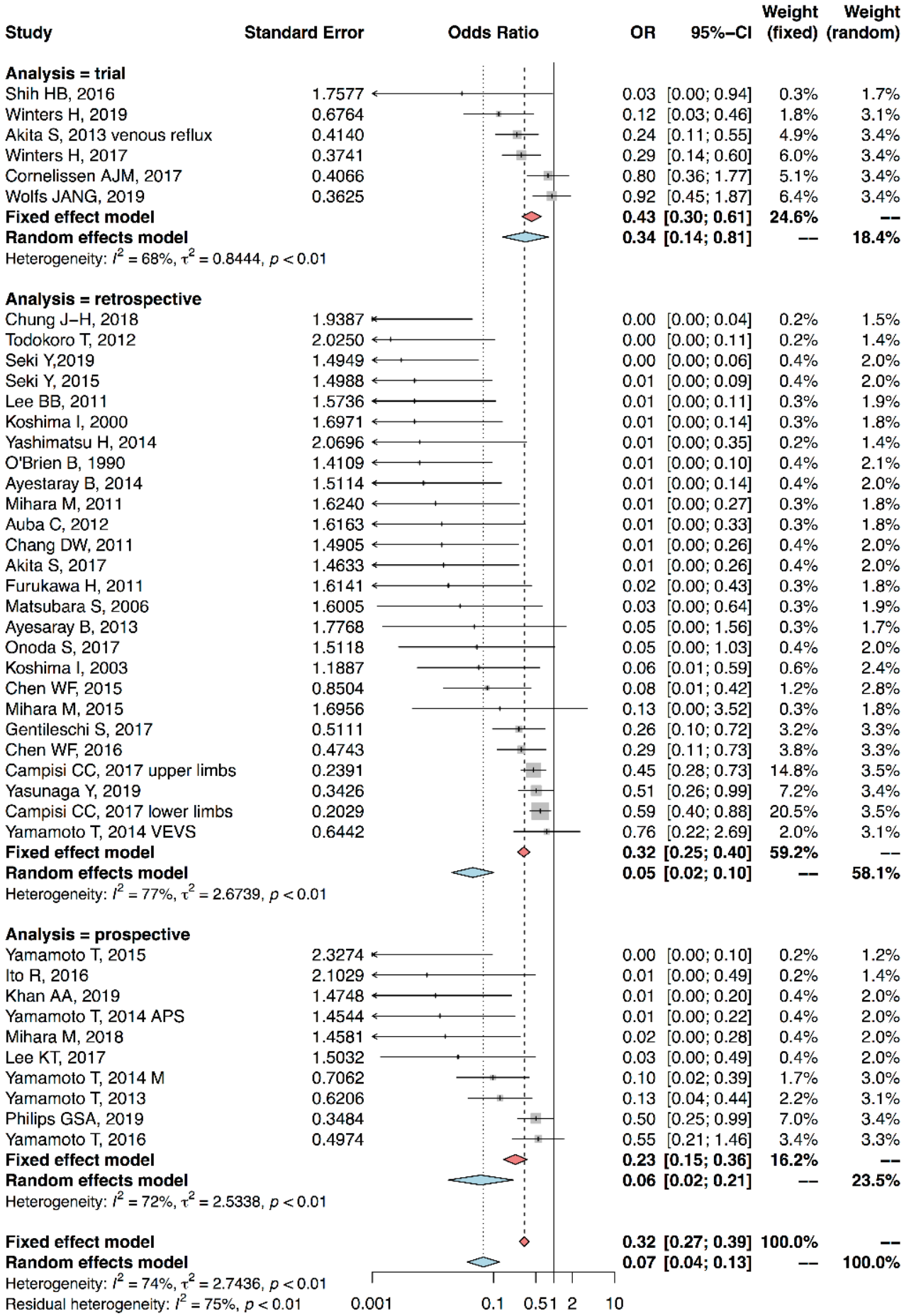

Fig. 2. Forest Plot of clinical trials and observational studies included in the meta-analysis examining the outcome efficacy of $L V A s$. 
Study

Seki Y, 2019

Chung J-H, 2018

Koshima I, 2000

Khan AA, 2019

O'Brien B, 1990

Chang DW, 2011

Shih HB, 2016

Akita S, 2017

Chen WF, 2016

Onoda S, 2017

Winters H, 2019

Lee KT, 2017

Gentileschi S, 2017

Winters H, 2017

Chen WF, 2015

Yamamoto T, 2014 VEVS

Campisi CC, 2017

Cornelissen AJM, 2017

Wolfs JANG, 2019

\section{Fixed effect model}

Random effects model

Heterogeneity: $I^{2}=70 \%, \tau^{2}=2.3804, p<0.01$

$$
0.001
$$

Weight Weight

Odds Ratio

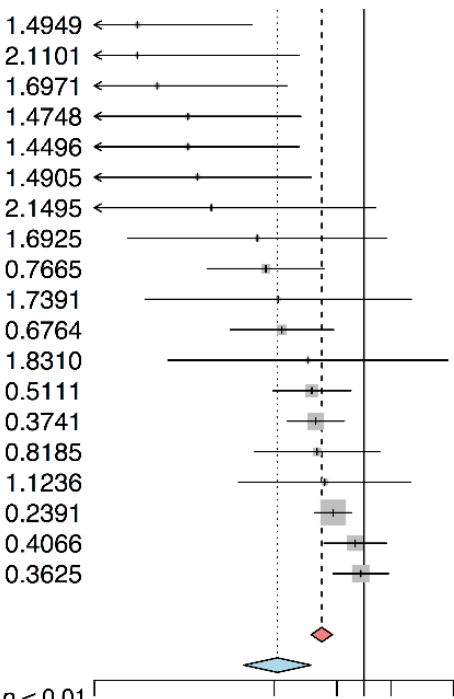

$\begin{array}{lll}0.1 & 0.512 \quad 10\end{array}$
OR $\quad 95 \%-\mathrm{Cl}$ (fixed) (random)

$0.00[0.00 ; 0.06] \quad 0.9 \%-4.2 \%$

$0.00[0.00 ; 0.19] \quad 0.4 \% \quad 2.8 \%$

$0.01 \quad[0.00 ; 0.14] \quad 0.7 \% \quad 3.7 \%$

$0.01[0.00 ; 0.20] \quad 0.9 \% \quad 4.2 \%$

$0.01[0.00 ; 0.19] \quad 0.9 \% \quad 4.3 \%$

$0.01[0.00 ; 0.26] \quad 0.9 \% \quad 4.2 \%$

$0.02[0.00 ; 1.35] \quad 0.4 \% \quad 2.8 \%$

$0.06[0.00 ; 1.79] \quad 0.7 \% \quad 3.7 \%$

$0.08[0.02 ; 0.36] \quad 3.3 \% \quad 6.5 \%$

$0.11[0.00 ; 3.35] \quad 0.6 \% \quad 3.6 \%$

$0.12[0.03 ; 0.46] \quad 4.2 \% \quad 6.8 \%$

$0.24[0.01 ; 8.61] \quad 0.6 \% \quad 3.4 \%$

$0.26[0.10 ; 0.72] \quad 7.4 \% \quad 7.3 \%$

$0.29[0.14 ; 0.60] \quad 13.8 \% \quad 7.7 \%$

$0.30[0.06 ; 1.49] \quad 2.9 \% \quad 6.3 \%$

$0.36[0.04 ; 3.30] \quad 1.5 \% \quad 5.3 \%$

$0.45[0.28 ; 0.73] \quad 33.7 \% \quad 7.9 \%$

$0.80[0.36 ; 1.77] \quad 11.7 \% \quad 7.6 \%$

$0.92[0.45 ; 1.87] \quad 14.7 \% \quad 7.7 \%$

0.34 [0.26; 0.45] $100.0 \%$ $0.11[0.05 ; 0.26] \quad-\quad 100.0 \%$
Study

Yamamoto T, 2015

Chung J-H, 2018

Seki Y, 2015

Lee BB, 2011

Yashimatsu H, 2014

Ito $\mathrm{R}, 2016$

Mihara M, 2011

Yamamoto T, 2014 APS

Mihara M, 2018

Akita S, 2017

Lee KT, 2017

Matsubara S, 2006

O'Brien B, 1990

Koshima I, 2003

Onoda S, 2017

Chen WF, 2016

Yamamoto T, $2014 \mathrm{M}$

Yamamoto T, 2013

Shih HB, 2016

Akita S, 2013 venous reflux

Yamamoto T, 2014 VEVS

Philips GSA, 2019

Yasunaga Y, 2019

Yamamoto T, 2016

Chen WF, 2015

Campisi CC, 2017
Standard Error

Odds Ratio

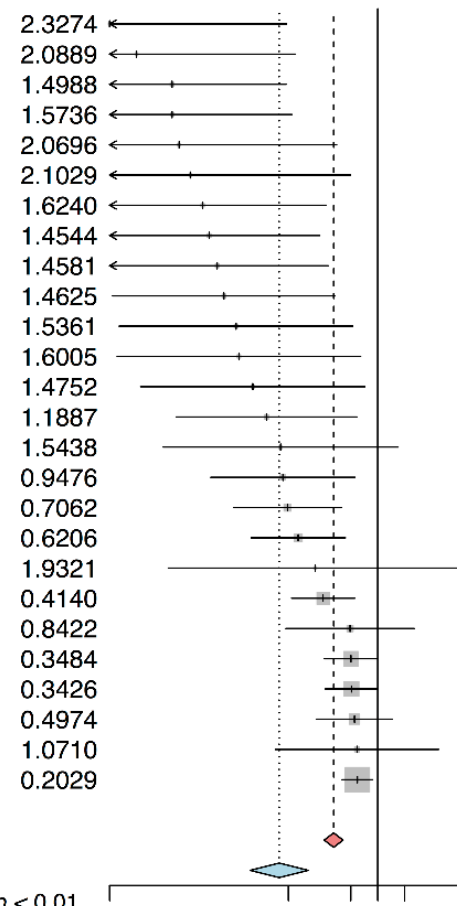

0.001

$\begin{array}{lll}0.1 & 0.512 \quad 10\end{array}$
Weight Weight OR $\quad 95 \%-\mathrm{Cl}$ (fixed) (random)

$0.00[0.00 ; 0.10] \quad 0.3 \% \quad 1.9 \%$ $0.00[0.00 ; 0.12] \quad 0.4 \% \quad 2.2 \%$

$0.01[0.00 ; 0.09] \quad 0.7 \% \quad 3.2 \%$

$0.01[0.00 ; 0.11] \quad 0.6 \% \quad 3.0 \%$

$0.01[0.00 ; 0.35] \quad 0.4 \% \quad 2.2 \%$

$0.01 \quad[0.00 ; 0.49] \quad 0.3 \% \quad 2.2 \%$

$0.01[0.00 ; 0.27] \quad 0.6 \% \quad 2.9 \%$

$0.01 \quad[0.00 ; 0.22] \quad 0.7 \% \quad 3.3 \%$

$0.02[0.00 ; 0.28] \quad 0.7 \% \quad 3.3 \%$

$0.02[0.00 ; 0.33] \quad 0.7 \% \quad 3.3 \%$

$0.03[0.00 ; 0.53] \quad 0.7 \% \quad 3.1 \%$

$0.03[0.00 ; 0.64] \quad 0.6 \% \quad 3.0 \%$

$0.04[0.00 ; 0.72] \quad 0.7 \% \quad 3.2 \%$

$0.06[0.01 ; 0.59] \quad 1.1 \% \quad 3.9 \%$

$0.08[0.00 ; 1.69] \quad 0.6 \% \quad 3.1 \%$

$0.09[0.01 ; 0.56] \quad 1.7 \% \quad 4.5 \%$

$0.10[0.02 ; 0.39] \quad 3.1 \% \quad 5.2 \%$

$0.13[0.04 ; 0.44] \quad 4.0 \% \quad 5.4 \%$

$0.20[0.00 ; 8.82] \quad 0.4 \% \quad 2.4 \%$

$0.24[0.11 ; 0.55] \quad 9.0 \% \quad 5.8 \%$

$0.49[0.09 ; 2.55] \quad 2.2 \% \quad 4.8 \%$

$0.50[0.25 ; 0.99] \quad 12.7 \% \quad 6.0 \%$

$0.51[0.26 ; 0.99] \quad 13.1 \% \quad 6.0 \%$

$0.55[0.21 ; 1.46] \quad 6.2 \% \quad 5.7 \%$

$0.59[0.07 ; 4.78] \quad 1.3 \% \quad 4.2 \%$

$0.59[0.40 ; 0.88] \quad 37.3 \% \quad 6.2 \%$ $\begin{array}{llr}0.32[0.25 ; 0.41] & 100.0 \% & -- \\ 0.08[0.04 ; 0.17] & - & 100.0 \%\end{array}$

Heterogeneity: $I^{2}=70 \%, \tau^{2}=2.3361, p<0.01$

\author{
.
}

Fig. 3. Forest Plot of subgroup analysis for studies of both upper (a) and lower (b) limb lymphedema examining the outcome efficacy of $L V A$. 
side anastomosis (I-square $=0 \%, \mathrm{OR}=\mathbf{0 . 0 2}$, $95 \% \mathrm{CI}=0.00-0.12, \mathrm{p}<0.01)$ testified the same efficacy (Fig. 4).

Information on the stage of pathology before surgery was available in 38 papers with 30 papers presenting patients affected by an early-moderate stage of pathology and the remaining 8 reporting cases of severe LE. The subgroup analysis resulted in similar efficacy for both groups with analysis using a random effect model (I-square $=69 \%)$ in patients with an early-moderate stage demonstrating an $\mathrm{OR}=0.11(95 \% \mathrm{CI}=0.06-0.23, \mathrm{p}<0.01)$, while the fixed model (I-square $=32 \%$ ) in patients with a severe stage showed an $\mathrm{OR}=0.21$ (95\% CI=0.07-0.61, p<0.01) (Fig. 5).

Similarly, the subgroup analysis on duration of pathology demonstrated the efficacy of LVAs in the treatment of LE independently from this variable. The fixed effect model (Isquare $=0 \%)$ showed an $\mathrm{OR}=0.06(95 \% \mathrm{CI}=$ $0.02-0.17, \mathrm{p}<0.01)$ in patients with a surgical treat-ment within 5 years from diagnosis, while the fixed effect model (I-square $=0 \%$ ) in patients treated from 5 to 10 year from diagnosis showed an $\mathrm{OR}=0.02(95 \% \mathrm{CI}=0.01-0.07$, $\mathrm{p}<0.01)$. In addition, subjects who had received microsurgical treatment after $\mathbf{1 0}$ years from diagnosis showed a fixed effect model $(\mathrm{I}$-square $=0 \%)$ of $\mathrm{OR}=0.02(95 \% \mathrm{CI}=$ 0.01-0.07, p<0.001) (Fig. 6).

\section{DISCUSSION}

We presented an updated overview and meta-analysis of the available literature about the efficacy of LVAs for the treatment of lymphedema. Although previous systematic reviews have been produced on this topic, the majority of them focused attention on specific site $(12,92)$ or specific etiology of lymphedema $(28,93)$. More consistent data are available for upper limb lymphedema secondary to breast cancer operation, and meta-analysis regarding effectiveness of LVAs in this pathology have been already performed. Results of these studies demonstrated a great heterogeneity of outcomes in volume/circumference reduction, while an improvement on quality of life, in particular in early-stage lymphedema, was confirmed (28). Less data are available for microsurgical treatment of lymphedema in other anatomical sites and studies on this topic highlighted the need for additional prospective studies $(\mathbf{9 2 , 9 4 , 9 5 ) \text { . }}$

The only comprehensive systematic review and meta-analysis concerning efficacy of micro-surgical techniques for the treatment of LE was performed by Basta et al (26). This study addressed the advantages and disadvantages of the various surgical techniques for the treatment of peripheral lymphedema, comparing results of 22 studies in which LVAs were used with 5 studies regarding lymph node transfers. This meta-analysis presented limitations related to the heterogeneity of the populations included into the subgroups as well as bias related to the comparison of lymphovenous shunt procedures with vascularized lymph node transfer and related to the inclusion into the lymphaticovenous anastomosis group, articles where lymph vessel transplantations were reported (96). Our meta-analysis included only studies reporting anastomosis between lymphatic and venous systems as a treatment for lymphedema. Subgroup analysis was performed to analyze more homogeneous populations, avoiding head-to-head comparison of different surgical procedures, different anatomical sites, and duration of pathology or stage of lymphedema.

Among the articles included in our metaanalysis, only 6 clinical trials were available, including a total of 144 patients. Four studies analyzed the efficacy of LVAs in secondary lymphedema in early or moderate stage of upper limbs using volumetry or UEL index as outcomes (29-32). Shih et al included 5 subjects with primary or secondary LE in moderate stage of upper or lower limbs (33), while Akita et al (34) compared the efficacy of sideto-end anastomosis alone and with vein valvuloplasty in lower limb lymphedema, showing results separately in patients with and without venous reflux at lymphography. Although meta-analysis results showed a statistically significant improvement of outcomes after performing LVAs, the low number of subjects included and the difference among studies related to follow-up, anatomical site, stage and duration of pathology, kind and 
TEC $=$ End to End Chung J-H, 2018

Seki Y,2019

Seki Y, 2015

Koshima I, 2000

Khan AA, 2019

Auba C, 2012

Mihara M, 2018

Lee KT, 2017

Shin HB, 2016

Koshima I, 2003

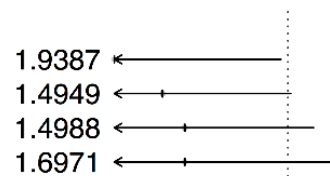

1.6971

1.4748

1.6163

1.4581

1.5032

1.7577

1.1887

Yamamoto T, 2016

0.4974

Cornelissen AJM, 2017

0.4066

Fixed effect model

Random effects model

Heterogeneity: $I^{2}=80 \%, \tau^{2}=2.9393, p<0.01$

TEC $=$ End to Side

Ayestaray B, 2014

Matsubara S, 2006

Ayestaray B, 2013

Fixed effect model

Random effects model

Heterogeneity: $I^{2}=0 \%, \tau^{2}=0.2006, p=0.68$

TEC $=$ MLVA

Campisi CC, 2017 UL

Campisi CC, 2017 LL

Fixed effect model

Random effects model

Heterogeneity: $I^{2}=0 \%, \tau^{2}=0.0093, p=0.40$

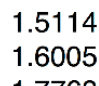

1.7768

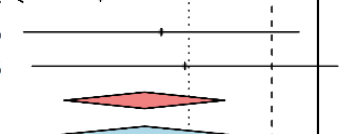

$0.01 \quad[0.00 ; 0.14]$

$0.03[0.00 ; 0.64]$

$0.05[0.00 ; 1.56]$

$0.02[0.00 ; 0.12]$

$0.02[0.00 ; 0.13]$

$0.6 \%$

$0.6 \%$

$0.5 \%$

$1.7 \%$

$3.8 \%$

$3.6 \%$

$3.3 \%$

$10.7 \%$

$0.45[0.28 ; 0.73] \quad 25.3 \%$

$6.4 \%$

$0.59[0.40 ; 0.88] \quad 35.2 \%$

$0.53[0.39 ; 0.72] \quad 60.5 \%$

$0.53[0.38 ; 0.74]$

$6.5 \%$

$12 . \overline{9} \%$
TEC = Side to End

Yamamoto T, 2015

Yashimatsu H, 2014

Yamamoto T, 2014 APS

Yamamoto T, 2013

Akita S, 2013 venous reflux

Yamamoto T, 2014 VEVS

Fixed effect model

Random effects model

Heterogeneity: $I^{2}=70 \%, \tau^{2}=4.2628, p<0.01$

\section{Fixed effect model}

Random effects model

Heterogeneity: $I^{2}=78 \%, \tau^{2}=3.1762, p<0.01$

Residual heterogeneity: $I^{2}=74 \%, p<0.01 \quad 0.001$

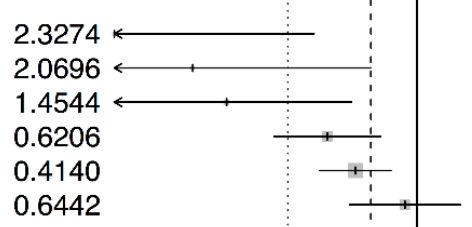

$0.00[0.00 ; 0.10]$

$0.01[0.00 ; 0.35]$

$0.01[0.00 ; 0.22]$

$0.3 \%$

$0.3 \%$

$0.7 \%$

$3.8 \%$

$0.24[0.11 ; 0.55] \quad 8.4 \%$

$0.76[0.22 ; 2.69] \quad 3.5 \%$

$0.20[0.11 ; 0.36] \quad 17.0 \%$

$0.07[0.01 ; 0.45]$

$0.35[0.28 ; 0.44] 100.0 \%$ $0.05[0.02 ; 0.13] \quad--100.0 \%$
$2.4 \%$

$2.8 \%$

$3.9 \%$

$5.8 \%$

$6.2 \%$

$5.8 \%$

$27.0 \%$

Fig. 4. Forest Plot of pooled results of all studies classified according to the surgical technique. The subgroup analysis reported an efficacy for all the tested procedures, either when using the fixed (end-to-end, side-to-end subgroups) or the random (MLVA, end-to-side) models. 
Stage $=$ Early moderate Todokoro T, 2012

Seki Y, 2019

Seki Y, 2015

Ito $\mathrm{R}, 2016$

Mihara M, 2011

Yamamoto T, 2014 APS

Auba C, 2012

Chang DW, 2011

Mihara M, 2018

Lee KT, 2017

Matsubara S, 2006

Shih HB, 2016

Onoda S, 2017

Chen WF, 2016

Winters H, 2019

\subsection{9}

1.4949

1.4988

2.1029

1.6240

1.4544

1.6163

1.4905

1.4581

1.5032

1.6005

1.7577

1.5118

0.6590

0.6764

0.6206

Yamamoto T, 2013

0.8820

0.5111

Gentileschi S, 2017

Winters $\mathrm{H}, 2017$

Yamamoto T, $2014 \mathrm{M}$

Yamamoto T, 2015

Philips GSA, 2019

Yasunaga Y, 2019

Yamamoto T, 2016

Chen WF, 2015

Cornelissen AJM, 2017

Wolfs JANG, 2019

Fixed effect model

Random effects model

Heterogeneity: $I^{2}=69 \%, \tau^{2}=2.4434, p<0.01$

0.3741

0.8613

0.8509

0.3484

0.3421

0.4974

0.7427

0.4066

0.3625
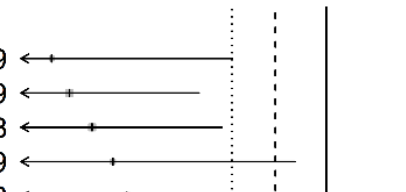

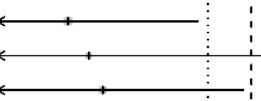

$0.00 \quad[0.00 ; 0.12]$

$0.00 \quad[0.00 ; 0.06]$

$0.01 \quad[0.00 ; 0.09]$

$0.01 \quad[0.00 ; 0.49]$

$0.01 \quad[0.00 ; 0.27]$

$0.01 \quad[0.00 ; 0.22]$

$0.01 \quad[0.00 ; 0.33]$

$0.01 \quad[0.00 ; 0.26]$

$0.02[0.00 ; 0.28]$

$0.03[0.00 ; 0.49]$

$0.03[0.00 ; 0.64]$

$0.03 \quad[0.00 ; 0.94]$

$0.05[0.00 ; 1.03]$

$0.11 \quad[0.03 ; 0.41]$

$0.12[0.03 ; 0.46]$

$0.13[0.04 ; 0.44]$

$0.19[0.03 ; 1.06]$

$0.26[0.10 ; 0.72]$

$0.29[0.14 ; 0.60]$

$0.40[0.07 ; 2.17]$

$0.45[0.08 ; 2.38]$

$0.50 \quad[0.25 ; 0.99]$

0.51 [0.26; 0.99$]$

$0.55[0.21 ; 1.46]$

$0.78[0.18 ; 3.34]$

$0.80[0.36 ; 1.77]$

$0.92[0.45 ; 1.87]$

$0.32[0.25 ; 0.41]$

0.11 [0.06; 0.23 ]

$0.3 \%$

$0.7 \%$

$0.7 \%$

$0.3 \%$

$0.6 \%$

$0.7 \%$

$0.6 \%$

$0.7 \%$

$0.7 \%$

$0.7 \%$

$0.6 \%$

$0.5 \%$

$0.7 \%$

$3.4 \%$

$3.3 \%$

$3.9 \%$

$1.9 \%$

$5.7 \%$

$10.7 \%$

$2.0 \%$

$2.1 \%$

$12.3 \%$

$12.8 \%$

$6.1 \%$

$2.7 \%$

$9.1 \%$

$11.4 \%$

$95.0 \%$

$--$
$1.7 \%$

$2.5 \%$

$2.5 \%$

$1.7 \%$

$2.3 \%$

$2.5 \%$

$2.3 \%$

$2.5 \%$

$2.5 \%$

$2.4 \%$

$2.3 \%$

$2.1 \%$

$2.4 \%$

$3.8 \%$

$3.8 \%$

$3.9 \%$

$3.5 \%$

$4.0 \%$

$4.2 \%$

$3.5 \%$

$3.5 \%$

$4.2 \%$

$4.2 \%$

$4.1 \%$

$3.7 \%$

$4.2 \%$

$4.2 \%$

$84.6 \%$

\section{Stage $=$ Severe}

Yamamoto T, 2015

Chen WF, 2016

Todokoro T, 2012

Yamamoto T, $2014 \mathrm{M}$

Poumellec MA, 2017

Chen WF, 2015

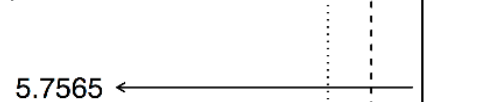

$0.00[0.00 ; 0.79] \quad 0.0 \%$

$0.3 \%$

1.0802

2.3115

$0.03[0.00 ; 0.26]$

$1.3 \%$

$3.1 \%$

$0.11[0.00 ; 10.30]$

1.4950

1.8310

1.0525

Yamamoto T, 2014 VEVS

1.2891

Fixed effect model

Random effects model

Heterogeneity: $I^{2}=32 \%, \tau^{2}=5.6732, p=0.18$

Fixed effect model

Random effects model

Heterogeneity: $I^{2}=64 \%, \tau^{2}=2.8076, p<0.01$

Residual heterogeneity: $l^{2}=65 \%, p<0.010 .001$

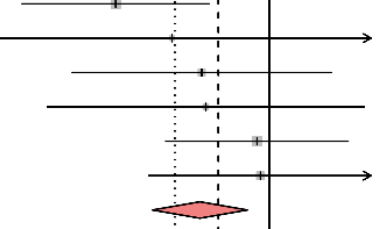

$0.22[0.01 ; 4.06]$

$0.3 \%$

$1.5 \%$

$0.24[0.01 ; 8.61] \quad 0.4 \%$

$2.5 \%$

$2.0 \%$

$0.76[0.10 ; 5.97] \quad 1.4 \% \quad 3.2 \%$

$0.82[0.07 ; 10.27] \quad 0.9 \% \quad 2.8 \%$

$0.21[0.07 ; 0.61] \quad 5.0 \%$

$\begin{array}{rrrr}0.21 & {[0.07 ; 0.61]} & 5.0 \% & - \\ 0.16 & {[0.02 ; 1.49]} & - & 15.4 \%\end{array}$

$0.32[0.25 ; 0.40] 100.0 \%$ $0.12[0.06 ; 0.24] \quad-\quad 100.0 \%$

Fig. 5. Forest Plot of the pooled results of all studies classified according to the stage of pathology as early, moderate, or severe. Analysis using a random effect model (I-square $=69 \%)$ in patients with an early-moderate stage showed an $\mathrm{OR}=0.11(95 \% \mathrm{CI}=0.06-0.23, p<0.01)$, while the fixed model (I-square=32\%) in patients with a severe stage showed a $O R=0.21(95 \% C I=0.07-0.61, p<0.01)$. This demonstrates efficacy of $L V A s$ in the treatment of LE is independent from the clinical stage of the lymphedema. 


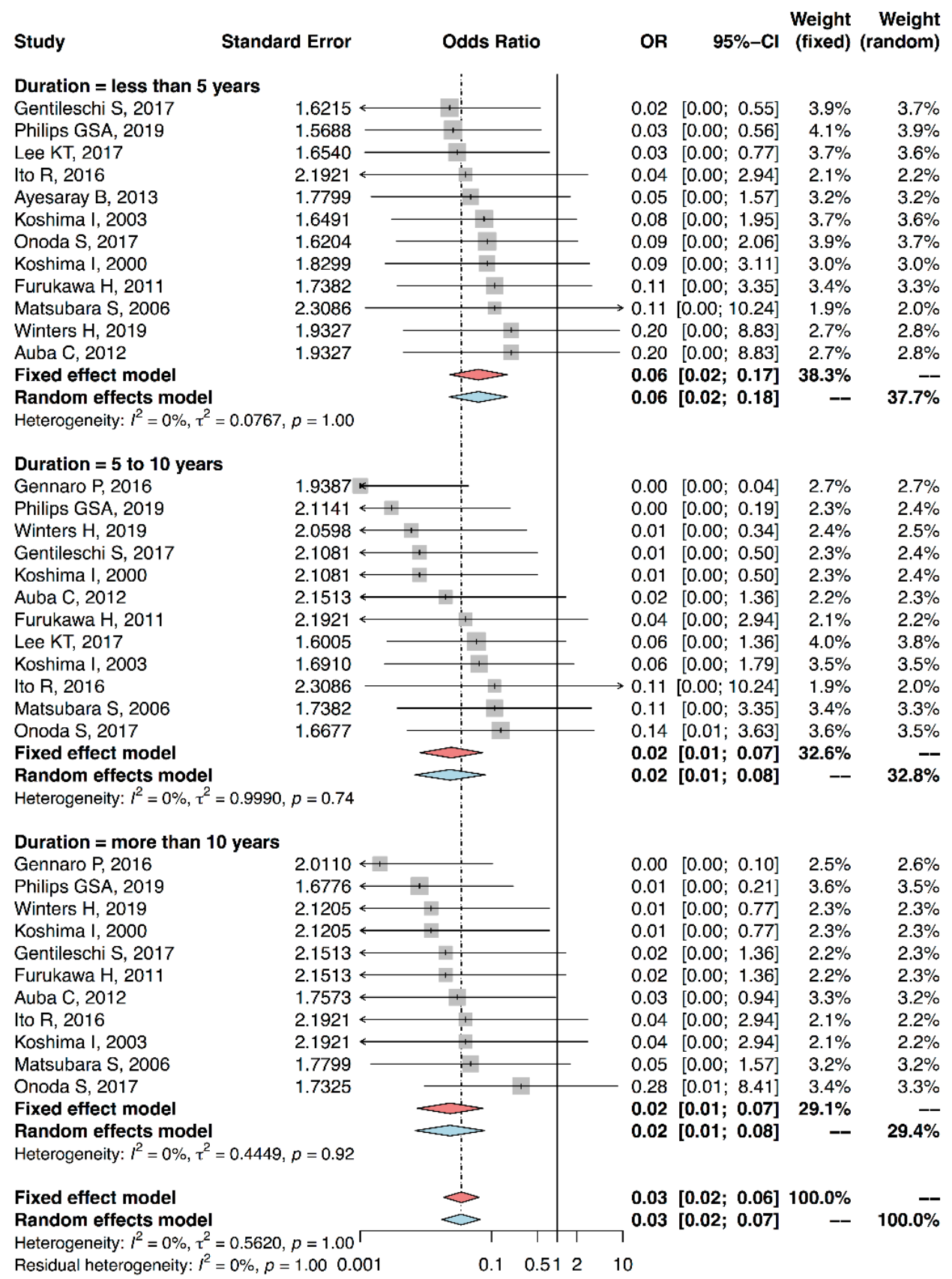

Fig. 6. Forest Plot of the pooled results of all studies classified according to the duration of the pathology for less than 5 years, from 5 to 10 years, and more than 10 years. Odds ratios are all smaller than one, with only some of the studies being not statistically significant at a 5\% level. Data exhibit small to no heterogeneity between each trial, making the fixed-effect model the best fit. Values of I-squared around 0 and p-values higher than 0.05 for each subgroup demonstrate that there is strong evidence for a uniform result. Subgroups are also pooled according to a fixed-effect model with the red diamond indicating a combined $O R=0.03$ with CI: $0.02-0.06$. This result is statistically significant, as 1 is not included in the $C I$. 
presentation (mean, difference of means, and percentage of subjects with positive outcomes) of endpoints did not allow an unbiased interpretation of results.

The inclusion of low risk of bias observational studies was performed to enlarge the cohort of study. MINOR index was used to select 42 high quality studies among 56 observational articles. The majority of studies confirmed a clear improvement in objective measurements, score of questionnaires or subjective symptoms after LVA, regardless of etiology, anatomical sites, surgical technique, stage or duration of LE. Results of the comprehensive meta-analysis performed in the overall cohort testified a strong efficacy of LVAs in treatment of LE, even if analysis was characterized by an important publication bias.

In relation to the anatomical field affected by LE, efficacy of LVAs was investigated in head and neck, pelvis, and scrotal lymphedema, but the presence of single studies on these topics did not allow the performance of a meta-analysis. On the contrary, a subgroup analysis for upper and lower limbs revealed a similar effectiveness of LVAs in treatment of lymphedema in both these fields, confirming the findings of several previous studies $(\mathbf{7 6 , 8 4 , 8 5 )}$.

Our investigation revealed great heterogeneity in the surgical procedure itself. The mean number of performed anastomosis ranged between 1 and 14.4, but there is no reported consensus on the optimal number of anastomoses required to yield a significant reduction in lymphedema. However, the importance of the number of anastomosis was debated: some authors emphasized its importance in lymphedema treatment $(8,41)$, while others did not specify the number of anastomoses, giving more importance to the adopted microsurgical technique $(53,76,97)$. For this reason, we preferred to perform a subgroup analysis according to the reported technique (end-to-end, end-to-side, side-to-end anastomosis or multiple-lymphatic-venous-anastomosis). Results of this analysis demonstrated efficacy of these surgical procedure in reduction of lymphedema.

Some authors reported a better effect of
LVAs in patients with low-moderate lymphedema (28), while our subgroup analysis on stage of lymphedema at diagnosis showed a statistically significant reduction of LE both in low-moderate and in severe lymphedema cases. Moreover, the efficacy of LVAs was demonstrated in a subgroup analysis independently from the duration of lymphedema.

The principal limitation of the current study is the risk of bias within studies. In fact, the level of evidence of the majority of studies was low, because of the lack of control groups in most studies (including trials) and the presence of a small sample size. True operative control groups are not possible or ethical for studies of LVA. Furthermore, information about inclusion of consecutive patients and experience of surgeons were often omitted, and some studies showed an insufficient follow-up period to evaluate long-term effect of LVAs. Thus, although we applied the MINOR index score to exclude the high risk of bias studies, the chance of selection bias could not be dismissed. Studies such as this metaanalysis on the LVA procedure in its current form is not something that is easily conducive to meta-analysis. There are many uncontrolled variables: primary vs secondary lymphedema, different severity of disease, varying surgeons' technical maturity/proficiency, varying quality of lymph vessels used to create LVA, varying number of LVA created per surgery, varying anastomotic configuration used in LVA, varying postoperative care, varying use of compression following surgery, and varying modalities used to track outcomes (volume, ICG lymphography, circumference measurement, bioimpedance), etc. Despite these limitations, improvement in subjective and/or objective outcomes was presented in the majority of the evaluated studies and a statistically significant effect of LVAs for the treatment of LE was demonstrated both in overall and in subgroup analysis. Specifically, effectiveness of LVAs was proven even when subgroup analysis was performed for stage, duration of pathology, anatomical site of lymphedema, or type of microsurgical procedure. Gaps among primary outcomes and heterogeneity in patients' characteristics 
reported into the studies could be a critical source of bias for a definitive confirmation of effectiveness of LVAs for the treatment of LE. Considering the potential for bias and limitations in study design, retrospective studies such as those reviewed represent the best information we currently have to investigate and show effectiveness for operations such as LVA.

\section{CONFLICT OF INTEREST AND DISCLOSURE}

The authors declare no competing financial interests exist.

\section{REFERENCES}

1. Brorson, H: Liposuction in Lymphedema Treatment. J. Reconstr. Microsurg. 32 (2016), 5665. doi:10.1055/s-0035-1549158

2. Ryan, TJ: Lymphatics and adipose tissue. Clin. Dermatol. 13 (1995), 493-498. doi:10.1016/0738081x(95)00092-t

3. Becker, C, J Assouad, M Riquet, G Hidden: Postmastectomy lymphedema: Long-term results following microsurgical lymph node transplantation. Ann. Surg. 243 (2006), 313-315. doi:10.1097/01.sla.0000201258.10304.16

4. Doscher. ME, S Herman, ES Garfein: Surgical management of inoperable lymphedema: The reemergence of abandoned techniques. J. Am. Coll. Surg. 215 (2012), 278-283.

doi:10.1016/j.jamcollsurg.2012.03.020

5. Giudice, G, F Robusto, M Vestita, et al: Singlestage excision and sentinel lymph node biopsy in cutaneous melanoma in selected patients: a retrospective case-control study. Melanoma Res. 27 (2017), 573-579. doi:10.1097/CMR.0000000000000370

6. Giudice, G, M Maruccia, E Nacchiero, et al: Dual plane breast implant reconstruction in large sized breasts: How to maximise the result following first stage total submuscular expansion. JPRAS Open. 15 (2018), 74-80. doi:10.1016/j.jpra.2017.11.003

7. The Diagnosis and Treatment of Peripheral Lymphedema: 2020 Consensus Document of the International Society of Lymphology. Lymphology 53 (2020), 3-19.

8. Chang, DW, H Suami, R Skoracki: A prospective analysis of 100 consecutive lymphovenous bypass cases for treatment of extremity lymphedema. Plast. Reconstr. Surg. 132 (2013), 1305-1314. doi:10.1097/PRS.0b013e3182a4d626

9. Campisi, C, F Boccardo: Microsurgical techniques for lymphedema treatment: Derivative lymphaticvenous microsurgery. World J. Surg. 28 (2004), 609-613. doi:10.1007/s00268-004-7252-4

10. Garza, R, R Skoracki, K Hock, SP Povoski: A comprehensive overview on the surgical management of secondary lymphedema of the upper and lower extremities related to prior oncologic therapies. BMC Cancer 17 (2017), 468. doi:10.1186/s12885-017-3444-9

11. Földi, E: The treatment of lymphedema. Cancer 83 (1998)(S12B), 2833-2834. doi:10.1002/(SICI)10970142(19981215)83:12B+<2833::AIDCNCR35>3.0.CO;2-3

12. Leung, N, D Furniss, H Giele: Modern surgical management of breast cancer therapy related upper limb and breast lymphoedema. Maturitas. 80 (2015), 384-390. doi:10.1016/j.maturitas.2015.01.012

13. Granzow, JW, JM Soderberg, AH Kaji, et al: Review of current surgical treatments for lymphedema. Ann. Surg. Oncol. 21 (2014), 11951201. doi:10.1245/s10434-014-3518-8

14. Maruccia, M, A Pezzolla, E Nacchiero, et al: Efficacy and early results after combining laparoscopic harvest of double gastroepiploic lymph node flap and active physiotherapy for lower extremity lymphedema. Microsurgery September 2019. doi:10.1002/micr.30511

15. Ciudad, P, A Mouchammed, OJ Manrique, et al: Comparison of long-term clinical outcomes among different vascularized lymph node transfers: 6-year experience of a single center's approach to the treatment of lymphedema. J. Surg. Oncol. January 2018. doi:10.1002/jso.24957

16. Maruccia, M, R Elia, $P$ Ciudad, et al: Postmastectomy upper limb lymphedema: Combined vascularized lymph node transfer and scar release with fat graft expedites surgical and patients' related outcomes. A retrospective comparative study. J. Plast. Reconstr. Aesthetic Surg. JPRAS. February 2019.

doi:10.1016/j.bjps.2019.01.029

17. Ciudad, $\mathbf{P}, \mathbf{M}$ Maruccia, J Socas, et al. The laparoscopic right gastroepiploic lymph node flap transfer for upper and lower limb lymphedema: Technique and outcomes. Microsurgery 37 (2017), 197-205. doi:10.1002/micr.22450

18. Di Taranto, G, R Elia, V Amorosi, et al: The difference in the caliber of efferent lymphatic vessels among various lymph node flaps. J. Surg. Oncol. 118 (2018), 1212-1213. doi:10.1002/jso.25257

19. Yamamoto, T, N Yamamoto, H Hara, et al: Upper extremity lymphedema index: A simple method for severity evaluation of upper extremity lymphedema. Ann. Plast. Surg. 70 (2013), 47-49. doi:10.1097/ SAP.0b013e3182275d23

20. Keeley, V, S Crooks, J Locke, et al: A quality of life measure for limb lymphoedema (LYMQOL). Clin. Res. 5 (2010), 13.

21. Chan, VSH, M Narushima, $\mathbf{H}$ Hara, et al: Local anesthesia for lymphaticovenular anastomosis. Ann. Plast. Surg. 72 (2014), 180-183. doi:10.1097/SAP.0b013e31825b3d1e

22. Ketterer, C: Surgical options for lymphedema following breast cancer treatment. Plast. Surg. Nurs. Off. J. Am. Soc. Plast. Reconstr. Surg. Nurses 
34 (2014), 82-85; quiz 86-87. doi:10.1097/PSN.0000000000000036

23. Leuzzi, S, M Maruccia, R Elia, et al: Lymphaticvenous anastomosis in a rat model: A novel exercise for microsurgical training. J. Surg. Oncol. 118 (2018), 936-940. doi:10.1002/jso.25234

24. Nacchiero, E, M Maruccia, M Vestita, et al: Multiple lymphatic-venous anastomoses in reducing the risk of lymphedema in melanoma patients undergoing complete lymph node dissection. A retrospective case-control study. J. Plast. Reconstr. Aesthetic Surg. JPRAS 72 (2019), 642-648. doi:10.1016/j.bjps.2019.01.023

25. Markkula, SP, N Leung, VB Allen, et al: Surgical interventions for the prevention or treatment of lymphoedema after breast cancer treatment. Cochrane Database Syst. Rev. 2 (2019), CD011433. doi:10.1002/14651858.CD011433.pub2

26. Basta, MN, LL Gao, LC Wu: Operative treatment of peripheral lymphedema: A systematic metaanalysis of the efficacy and safety of lymphovenous microsurgery and tissue transplantation. Plast. Reconstr. Surg. 133 (2014), 905-913. doi:10.1097/PRS.0000000000000010

27. Slim, K, E Nini, D Forestier: Methodological index for non-randomized studies (minors): Development and validation of a new instrument. ANZ J. Surg. 73 (2003), 712-716. doi:10.1046/j.14452197.2003.02748.x

28. Cornelissen, AJM, J Beugels, L Ewalds, et al. Effect of lymphaticovenous anastomosis in breast cancer-related lymphedema: A review of the literature. Lymphat. Res. Biol. 16 (2018), 426-434. doi:10.1089/lrb.2017.0067

29. Winters, H, HJP Tielemans, AC Verhulst, et al: The Long-term Patency of Lymphaticovenular anastomosis in breast cancer-related lymphedema. Ann Plast Surg. 82 (2019), 196-200. doi:10.1097/SAP.0000000000001674

30. Wolfs, JAGN, LGEH de Joode, RRWJ van der Hulst, et al: Correlation between patency and clinical improvement after lymphaticovenous anastomosis (LVA) in breast cancer-related lymphedema: 12-month follow-up. Breast Cancer Res. Treat. 179 (2020), 131-138. doi:10.1007/s10549-019-05450-2

31. Winters, H, HJP Tielemans, M Hameeteman, et al: The efficacy of lymphaticovenular anastomosis in breast cancer-related lymphedema. Breast Cancer Res. Treat. 165 (2017), 321-327. doi:10.1007/s10549-017-4335-0

32. Cornelissen, AJM, M Kool, TR Lopez Penha, et al: Lymphatico-venous anastomosis as treatment for breast cancer-related lymphedema: A prospective study on quality of life. Breast Cancer Res. Treat. 163 (2017), 281-286. doi:10.1007/s10549-017-4180-1

33. Shih, HB, A Shakir, DH Nguyen: Use of Indocyanine Green-SPY angiography for tracking lymphatic recovery after lymphaticovenous anastomosis. Ann. Plast. Surg. 76 (2016)(Suppl 3), S232-237. doi:10.1097/SAP.0000000000000766

34. Akita, S, N Mitsukawa, M Kuriyama, et al:
External valvuloplasty for subcutaneous small veins to prevent venous reflux in lymphaticovenular anastomosis for lower extremity lymphedema. Plast. Reconstr. Surg. 132 (2013), 1008-1014. doi:10.1097/PRS.0b013e31829fe12f

35. de Vos-Kerkhof, E, DHF Geurts, M Wiggers, et al: Tools for "safety netting" in common paediatric illnesses: A systematic review in emergency care. Arch. Dis. Child. 101 (2016), 131-139. doi:10.1136/archdischild-2014-306953

36. Suzuki, Y, H Sakuma, S Yamazaki: Comparison of patency rates of lymphaticovenous anastomoses at different sites for lower extremity lymphedema. J. Vasc. Surg. Venous Lymphat. Disord. 7 (2019), ;7(2):222-227. doi:10.1016/j.jvsv.2018.10.022

37. Gentileschi, S, R Albanese, V Pino, et al: SPECT/CT and fusion ultrasound to target the efferent groin lymph node for lymphatic surgery. Microsurgery 39 (2019), 605-612.

doi:10.1002/micr.30501

38. Salgarello, M, ML Mangialardi, V Pino, et al: A prospective evaluation of health-related quality of life following lymphaticovenular anastomosis for upper and lower extremities lymphedema. $\mathbf{J}$ Reconstr. Microsurg. 34 (2018), 701-707. doi:10.1055/s-0038-1642623

39. Hara, H, M Mihara: Lymphaticovenous anastomosis and resection for genital acquired lymphangiectasia (GAL). J. Plast. Reconstr. Aesthetic Surg. JPRAS 71 (2018), 1625-1630. doi:10.1016/j.bjps.2018.05.015

40. Engel, H, C-Y Lin, JJ Huang, et al: Outcomes of lymphedema microsurgery for breast cancerrelated lymphedema with or without microvascular breast reconstruction. Ann. Surg. 268 (2018), 10761083. doi:10.1097/SLA.0000000000002322

41. Mihara, M, H Hara, S Tange, et al: Multisite lymphaticovenular bypass using supermicrosurgery technique for lymphedema management in lower lymphedema cases. Plast. Reconstr. Surg. 138 (2016), 262-272. doi:10.1097/PRS.0000000000002254

42. Torrisi, JS, WJ Joseph, S Ghanta, et al: Lymphaticovenous bypass decreases pathologic skin changes in upper extremity breast cancerrelated lymphedema. Lymphat. Res. Biol. 13 (2015), 46-53. doi:10.1089/lrb.2014.0022

43. Damstra, RJ, HGJ Voesten, WD van Schelven, et al: Lymphatic venous anastomosis (LVA) for treatment of secondary arm lymphedema. A prospective study of $11 \mathrm{LVA}$ procedures in $\mathbf{1 0}$ patients with breast cancer related lymphedema and a critical review of the literature. Breast Cancer Res. Treat. 113 (2009), 199-206. doi:10.1007/s10549-008-9932-5

44. Campisi, C, F Boccardo, A Zilli, et al: Long-term results after lymphatic-venous anastomoses for the treatment of obstructive lymphedema.

Microsurgery 21 (2001), 135-139. doi:10.1002/micr.1025

45. Ipsen, T, J Pless, PB Frederiksen: Experience with microlymphaticovenous anastomoses for congenital 
and acquired lymphoedema. Scand. J. Plast. Reconstr. Surg. Hand Surg. 22 (1988), 233-236. doi:10.3109/02844318809097950

46. Gloviczki, P, J Fisher, LH Hollier, et al: Microsurgical lymphovenous anastomosis for treatment of lymphedema: A critical review. J. Vasc. Surg. 7 (1988), 647-652. doi:10.1067/mva.1988.avs0070647

47. Struick van Bemmelen, SP, GA Olthuis, RJ Dinkelman: Reconstructive approach of chronic oedema of the lower limb. Neth. J. Surg. 40 (1988), 64-68.

48. Huang, GK, RQ Hu, ZZ Liu, et al: Microlymphaticovenous anastomosis in the treatment of lower limb obstructive lymphedema: Analysis of 91 cases. Plast. Reconstr. Surg. 76 (1985), 671-685.

49. Degni, M: New technique of lymphatic-venous anastomosis for the treatment of lymphedema. $\mathbf{J}$. Cardiovasc. Surg. (Torino). 19 (1978), 577-580.

50. Ayestaray, B, F Bekara, J-B Andreoletti: $\pi$-shaped lymphaticovenular anastomosis for head and neck lymphoedema: A preliminary study. J. Plast. Reconstr. Aesthetic Surg. JPRAS. 66 (2013), 201206. doi:10.1016/j.bjps.2012.08.049

51. Todokoro, T, D Furniss, K Oda, et al: Effective treatment of pelvic lymphocele by lymphaticovenular anastomosis. Gynecol. Oncol. 128 (2013), 209-214. doi:10.1016/j.ygyno.2012.11.014

52. Mukenge, S, D Negrini, M Catena, et al: Development of functionally patent lymphatic meshes in postsurgical long-term resolution of peripheral secondary lymphedema. J. Vasc. Surg. Venous Lymphat. Disord. 1 (2013), 280-288. doi:10.1016/j.jvsv.2012.09.008

53. Koshima, I, Y Nanba, T Tsutsui, et al: Long-term follow-up after lymphaticovenular anastomosis for lymphedema in the leg. J. Reconstr. Microsurg. 19 (2003), 209-215. doi:10.1055/s-2003-40575

54. Koshima, I, K Inagawa, K Urushibara, et al: Supermicrosurgical lymphaticovenular anastomosis for the treatment of lymphedema in the upper extremities. J. Reconstr. Microsurg. 2000;16(6):437-442. doi:10.1055/s-2006-947150

55. Poumellec, M-A, R Foissac, M Cegarra-Escolano, et al: Surgical treatment of secondary lymphedema of the upper limb by stepped microsurgical lymphaticovenous anastomoses. Breast Cancer Res. Treat. 162 (2017), 219-224. doi:10.1007/s10549-0174110-2

56. Gennaro, P, G Gabriele, M Mihara, et al: Supramicrosurgical lymphatico-venular anastomosis (LVA) in treating lymphoedema: 36months preliminary report. Eur. Rev. Med. Pharmacol. Sci. 20 (2016), 4642-4653.

57. Chen. WF, H Zhao, T Yamamoto, et al: Indocyanine green lymphographic evidence of surgical efficacy following microsurgical and supermicrosurgical lymphedema reconstructions. J. Reconstr. Microsurg. 32 (2016), 688-698. doi:10.1055/s-0036-1586254
58. Chen, WF, T Yamamoto, M Fisher, et al: The "Octopus" lymphaticovenular anastomosis: evolving beyond the standard supermicrosurgical technique. J. Reconstr. Microsurg. 31 (2015), 450-457. doi:10.1055/s-0035-1548746

59. Yamamoto, T, H Yoshimatsu, N Yamamoto, et al: Multisite lymphaticovenular anastomosis using vein graft for uterine cancer-related lymphedema after pelvic lymphadenectomy. Vasc. Endovascular Surg. 49 (2015), 195-200. doi:10.1177/1538574415614402

60. Yamamoto, T, N Yamamoto, T Numahata, et al: Navigation lymphatic supermicrosurgery for the treatment of cancer-related peripheral lymphedema. Vasc. Endovascular Surg. 48 (2014), 139-143. doi:10.1177/1538574413510979

61. Yamamoto, T, N Yamamoto, S Azuma, et al: Nearinfrared illumination system-integrated microscope for supermicrosurgical lymphaticovenular anastomosis. Microsurgery 34 (2014), 23-27. doi:10.1002/micr.22115

62. Ayestaray, B, F Bekara: $\pi$-shaped lymphaticovenular anastomosis: The venous flow sparing technique for the treatment of peripheral lymphedema. J. Reconstr. Microsurg. 30 (2014), 551-560. doi:10.1055/s-0034-1370356

63. Matsubara, S,H Sakuda, M Nakaema, et al: Longterm results of microscopic lymphatic vesselisolated vein anastomosis for secondary lymphedema of the lower extremities. Surg. Today. 36 (2006), 859-864. doi:10.1007/s00595-006-3269-7

64. Campisi, CC, M Ryan, F Boccardo, et al: Fibrolipo-lymph-aspiration with a lymph vessel sparing procedure to treat advanced lymphedema after multiple lymphatic-venous anastomoses: The complete treatment protocol. Ann. Plast. Surg. 78 (2017), 184-190. doi:10.1097/SAP.0000000000000853

65. Boccardo, F, F De Cian, CC Campisi, et al: Surgical prevention and treatment of lymphedema after lymph node dissection in patients with cutaneous melanoma. Lymphology 46 (2013), 2026.

66. Akita, S, F Ogata, I Manabe, et al: Noninvasive screening test for detecting early stage lymphedema using follow-up computed tomography imaging after cancer treatment and results of treatment with lymphaticovenular anastomosis. Microsurgery 37 (2017), 910-916. doi:10.1002/micr.30188

67. Onoda, S, K Yamada, K Matsumoto, et al: A detailed examination of the characteristics and treatment in a series of 33 idiopathic lymphedema patients. J. Reconstr. Microsurg. 33 (2017), 19-25. doi:10.1055/s-0036-1586257

68. Furukawa, H, M Osawa, A Saito, et al: Microsurgical lymphaticovenous implantation targeting dermal lymphatic backflow using indocyanine green fluorescence lymphography in the treatment of postmastectomy lymphedema. Plast. Reconstr. Surg. 127 (2011), 1804-1811. doi:10.1097/PRS.0b013e31820cf2e2

69. Mihara, M, Y Hayashi, N Murai, et al: Regional 
diagnosis of lymphoedema and selection of sites for lymphaticovenular anastomosis using elastography. Clin. Radiol. 66 (2011), 715-719.

doi:10.1016/j.crad.2011.03.004

70. Chang, DW: Lymphaticovenular bypass for lymphedema management in breast cancer patients: A prospective study. Plast. Reconstr. Surg. 126 (2010), 752-758. doi:10.1097/PRS.0b013e3181e5f6a9

71. Phillips, GSA, S Gore, A Ramsden, et al: Lymphaticovenular anastomosis in the treatment of secondary lymphoedema of the legs after cancer treatment. J. Plast. Reconstr. Aesthetic Surg. JPRAS 72 (2019), 1184-1192. doi:10.1016/j.bjps.2019.03.013

72. Yasunaga, Y, D Yanagisawa, E Ohata, et al: Bioelectrical impedance analysis of water reduction in lower-limb lymphedema by lymphaticovenular anastomosis. J. Reconstr. Microsurg. 35 (2019), 306-314. doi:10.1055/s-0038-1675368

73. Gentileschi, S, M Servillo, R Albanese, et al: Lymphatic mapping of the upper limb with lymphedema before lymphatic supermicrosurgery by mirroring of the healthy limb. Microsurgery 37 (2017), 881-889. doi:10.1002/micr.30247

74. Ito, R, C-T Wu, MC-Y Lin, et al: Successful treatment of early-stage lower extremity lymphedema with side-to-end lymphovenous anastomosis with indocyanine green lymphography assisted. Microsurgery 36 (2016), 310-315. doi:10.1002/micr.30010

75. Lee, BB, J Laredo, R Neville: Reconstructive surgery for chronic lymphedema: A viable option, but. Vascular 19 (2011), 195-205. doi:10.1258/vasc.2010.0a0287

76. O'Brien, BM, CG Mellow, RK Khazanchi, et al: Long-term results after microlymphaticovenous anastomoses for the treatment of obstructive lymphedema. Plast. Reconstr. Surg. 85 (1990), 562572. doi:10.1097/00006534-199004000-00011

77. Seki, Y, A Kajikawa, T Yamamoto, et al: The dynamic-lymphaticovenular anastomosis method for breast cancer treatment-related lymphedema: Creation of functional lymphaticovenular anastomoses with use of preoperative dynamic ultrasonography. J. Plast. Reconstr Aesthetic Surg. JPRAS 72 (2019), 62-70. doi:10.1016/j.bjps.2018.09.005

78. Khan, AA, I Hernan, JA Adamthwaite, et al: Feasibility study of combined dynamic imaging and lymphaticovenous anastomosis surgery for breast cancer-related lymphoedema. Br. J. Surg. 106 (2019), 100-110. doi:10.1002/bjs.10983

79. Chung, J-H, S-O Baek, H-J Park, et al: Efficacy and patient satisfaction regarding lymphovenous bypass with sleeve-in anastomosis for extremity lymphedema. Arch. Plast. Surg. 46 (2019), 46-56. doi:10.5999/aps.2018.00773

80. Lee, K-T, J-W Park, G-H Mun: Serial two-year follow-up after lymphaticovenular anastomosis for the treatment of lymphedema. Microsurgery 37 (2017), 763-770. doi:10.1002/micr.30200
81. Seki, Y, T Yamamoto, $H$ Yoshimatsu, et al: The Superior-Edge-of-the-Knee incision method in lymphaticovenular anastomosis for lower extremity lymphedema. Plast. Reconstr. Surg. 136 (2015), 665e-675e. doi:10.1097/PRS.0000000000001715

82. Mihara, M, H Hara, J Shibasaki, et al: Indocyanine green lymphography and lymphaticovenous anastomosis for generalized lymphatic dysplasia with pleural effusion and ascites in neonates. Ann. Vasc. Surg. 29 (2015), 1111-1122. doi:10.1016/j.avsg.2015.02.013

83. Auba, C, D Marre, G Rodríguez-Losada, et al: Lymphaticovenular anastomoses for lymphedema treatment: 18 months postoperative outcomes. Microsurgery 32 (2012), 261-268. doi:10.1002/micr.20980

84. Demirtas, Y, N Ozturk, O Yapici, et al: Comparison of primary and secondary lowerextremity lymphedema treated with supermicrosurgical lymphaticovenous anastomosis and lymphaticovenous implantation. J. Reconstr. Microsurg. 26 (2010), 137-143. doi:10.1055/s-00291243299

85. Koshima, I, Y Nanba, T Tsutsui, et al: Minimal invasive lymphaticovenular anastomosis under local anesthesia for leg lymphedema: Is it effective for stage III and IV? Ann. Plast. Surg. 53 (2004), 261-266. doi:10.1097/01.sap.0000120319.89926.07

86. Mihara, M, H Hara, Y Kawakami, et al: Multi-site lymphatic venous anastomosis using echography to detect suitable subcutaneous vein in severe lymphedema patients. J. Plast. Reconstr. Aesthetic Surg. JPRAS 71 (2018), e1-e7. doi:10.1016/j.bjps.2017.10.004

87. Seki, Y, A Kajikawa, T Yamamoto, et al: Single lymphaticovenular anastomosis for early-stage lower extremity lymphedema treated by the superior-edge-of-the-knee incision method. Plast. Reconstr. Surg. Glob. Open. 6 (2018), e1679. doi:10.1097/GOX.0000000000001679

88. Yamamoto, T, N Yamamoto, M Yamashita, et al: Efferent lymphatic vessel anastomosis: Supermicrosurgical efferent lymphatic vessel-tovenous anastomosis for the prophylactic treatment of subclinical lymphedema. Ann. Plast. Surg. 76 (2016), 424-427. doi:10.1097/SAP.0000000000000381

89. Yoshimatsu, H, T Yamamoto, M Narushima, et al: The guide wire method: a new technique for easier side-to-end lymphaticovenular anastomosis. Ann. Plast. Surg. 73 (2014), 231-233. doi:10.1097/SAP.0b013e318276d99a

90. Yamamoto, T, H Yoshimatsu, M Narushima, et al: Sequential anastomosis for lymphatic supermicrosurgery: multiple lymphaticovenular anastomoses on 1 venule. Ann. Plast. Surg. 73 (2014), 46-49. doi:10.1097/SAP.0b013e31826caff1

91. Yamamoto, T, H Yoshimatsu, M Narushima, et al: A modified side-to-end lymphaticovenular anastomosis. Microsurgery 33 (2013), 130-133. doi:10.1002/micr.22040

92. Aulia, I, EC Yessica: Surgical management of male 
genital lymphedema: A systematic review. Arch. Plast. Surg. 47 (2020), 3-8. doi:10.5999/aps.2019.01123

93. Kanda, MH, RA da Costa Vieira, JPSN Lima, CE Paiva, et al: Late locoregional complications associated with adjuvant radiotherapy in the treatment of breast cancer: Systematic review and meta-analysis. J. Surg. Oncol. December 2019. doi:10.1002/jso.25820

94. Dessources, K, E Aviki, MM Leitao: Lower extremity lymphedema in patients with gynecologic malignancies. Int. J. Gynecol. Cancer Off. J. Int. Gynecol. Cancer Soc. 30 (2020), 252-260. doi:10.1136/ijgc-2019-001032

95. Abbas, S, M Seitz: Systematic review and metaanalysis of the used surgical techniques to reduce leg lymphedema following radical inguinal nodes dissection. Surg. Oncol. 20 (2011), 88-96.

doi:10.1016/j.suronc.2009.11.003
96. Zeltzer, AA, M Hamdi: Operative treatment of peripheral lymphedema: A systematic metaanalysis of the efficacy and safety of lymphovenous microsurgery and tissue transplant. Plast. Reconstr. Surg. 134 (2014), 491e-492e. doi:10.1097/PRS.0000000000000441

97. Szuba, A, SG Rockson: Lymphedema: Classification, diagnosis and therapy. Vasc. Med. Lond. Engl. 3 (1998), 145-156. doi:10.1177/1358836X9800300209

Rossella Elia, M.D.

Piazza Giulio Cesare 11

Bari, 70124, Italy

Phone: +393409616476

E-mail: rossellaelia4@gmail.com 